Article

\title{
Reliability Enhancement in Power Networks under Uncertainty from Distributed Energy Resources ${ }^{\dagger}$
}

\author{
Mike Brian Ndawula ${ }^{1, *}\left(\mathbb{D}\right.$, Sasa Z. Djokic ${ }^{2}(\mathbb{D}$ and Ignacio Hernando-Gil $1,3(\mathbb{0}$ \\ 1 Centre for Sustainable Power Distribution, Department of Electronic and Electrical Engineering, \\ University of Bath, Bath BA2 7AY, UK; i.hernando.gil@bath.ac.uk \\ 2 Institute for Energy Systems, University of Edinburgh, King's Buildings, Mayfield Road, \\ Edinburgh EH9 3JL, UK; sasa.djokic@ed.ac.uk \\ 3 ESTIA Institute of Technology, University of Bordeaux, F-64210 Bidart, France \\ * Correspondence: m.b.ndawula@bath.ac.uk \\ $+\quad$ This work is an extension of the paper, "Impact of the stochastic behaviour of distributed energy resources \\ on MV/LV network reliability", presented at the 18th IEEE International Conference on Environment and \\ Electrical Engineering (EEEIC/I\&CPS Europe), Palermo, Italy, 12-15 June 2018.
}

Received: 21 December 2018; Accepted: 1 February 2019; Published: 7 February 2019

\begin{abstract}
This paper presents an integrated approach for assessing the impact that distributed energy resources (DERs), including intermittent photovoltaic (PV) generation, might have on the reliability performance of power networks. A test distribution system, based on a typical urban MV and LV networks in the UK, is modelled and used to investigate potential benefits of the local renewable generation, demand-manageable loads and coordinated energy storage. The conventional Monte Carlo method is modified to include time-variation of electricity demand profiles and failure rates of network components. Additionally, a theoretical interruption model is employed to assess more accurately the moment in time when interruptions to electricity customers are likely to occur. Accordingly, the impact of the spatio-temporal variation of DERs on reliability performance is quantified in terms of the effect of network outages. The potential benefits from smart grid functionalities are assessed through both system- and customer-oriented reliability indices, with special attention to energy not supplied to customers, as well as frequency and duration of supply interruptions. The paper also discusses deployment of an intelligent energy management system to control local energy generation-storage-demand resources that can resolve uncertainties in renewable-based generation and ensure highly reliable and continuous supply to all connected customers.
\end{abstract}

Keywords: demand profiles; demand response; distributed (PV) generation; energy storage; failure rate; Monte Carlo simulation; network reliability; renewable resources

\section{Introduction}

The "smart grid" concept generally denotes a flexible electricity network, capable of providing different services and functionalities, such as improved control and balancing of power flows, or enhanced fault protection. An important aspect of smart grid is the use of distributed generation (DG), which should be controlled to ensure higher energy efficiency and maximum asset utilisation [1]. The paradigm shift to smart grids is driven by the fact that existing/legacy networks require high investments to meet various techno-economic requirements of the increasingly dynamic energy landscape. Within this context, smart grid technologies include demand side response (DSR), energy storage (ES) and microgeneration (MG), collectively termed as distributed energy resources (DERs), 
as well as enhancement of enabling infrastructures for monitoring, communication and control of network assets. Successful management of these smart grid technologies is expected to result in higher energy efficiency and supply security, thus allowing for deferral of investments in network reinforcement, upgrading and expansion [2]. Due to requirements for supply sustainability, renewable energy resources (RERs) will be instrumental in realising changes to planning and operation of power systems, culminating in significant economic and environmental benefits. These benefits include provision of not only residential low-voltage (LV) customers, but also commercial and industrial medium-voltage (MV) customers, with the capacity to reduce their electricity consumption and energy bills, plus participate in ancillary service markets through the provision of frequency response services and short-term operating reserves [3].

In realising the significant benefits of smart grid technologies, it is important to overcome the reliance on the intermittent outputs of RERs and resolve uncertainties in network operation introduced by their stochastic behaviour [4]. For example, the times of the peak photovoltaic (PV) power generation might not, and typically do not coincide with the peak demands (depending on the category of end consumer), which results in a less than optimal utilisation of the generated PV power [5]. The deployment of RER must therefore be carefully analysed in terms of their impacts on the network performance, quality of supply (QS) delivered to end customers, network reliability and resulting financial implications, e.g., potential for creation of peer-to-peer energy trading and ancillary service markets. In that context, the impact of DERs on supply interruptions is particularly important as these traditionally have the highest social and economic implications to all stakeholders in the electricity sector. Accordingly, regulators enforce legislation to make sure that the highest standards of network reliability performance and supply continuity are provided to the electricity customers, e.g., through the security and quality of supply (SQS) legislation in the UK [6,7]. To correctly quantify the impact of different DER technologies on network reliability performance, distribution network operators (DNOs) commonly use a set of standard indices [8] to measure system reliability and must report these to regulatory bodies as a measure of their performance.

This paper builds on the previous work published in [4] and [9], where the impact from the implementation of ES, renewable-based MG systems and DSR on actively managed distribution networks was analysed. The analysis in this paper acknowledges that solar PV power production is strongly impacted by unpredictable cloud movements and thus presents PV models that can evaluate related temporal and spatial variations to correctly quantify potential benefits. In published literature, different methods have been proposed to mitigate the power output fluctuations due to this 'clouding' effect, such as ramp-rate control using ES [10]. In the previous work, compensation of PV fluctuations using ES to achieve flat feeder voltage profiles, to reduce number of voltage-regulation operations and to lower system operational costs is discussed in [11]. Similarly, [12] discusses quantification of the effects of PV on power system reliability, but mainly focuses on finding the optimal placement of PV installations and employs a relatively low resolution time-step (1 day) for the presented probabilistic models. Finally, [13] focuses on minimizing lifetime, energy and load-loss costs. Despite these developments, there is a need to more accurately quantify the impact of these stochastically-behaving local PV solutions on reliability performance and assess their direct implications on supply continuity and, importantly, customer "willingness-to-pay".

In order to address some of the above challenges, part of the contribution of this paper is to present a more realistic PV modelling, in order to quantify clouding effects in reliability studies. Furthermore, the paper presents a general methodology for the optimal design of an energy management system (EMS), to control ES and realise an improved reliability performance. The importance of ES is highlighted in several studies assessing the benefits from ES for peak shaving, frequency regulation and operational optimisation, especially when the ES is installed in a centralised configuration, which is usually a preferred option by the DNOs [14-16]. Although there are various studies that focus on ES sizing, economic operation and optimization [17], less emphasis is put on the technical capabilities of ES for devising smart functionalities to improve overall network reliability performance. In this 
paper, ES systems are modelled in a highly distributed configuration, i.e. as the small-size ES operating together with the local PV-based MG units. This is assumed to be a typical smart grid configuration in e.g., residential applications, and this paper demonstrates that these applications can be designed and implemented to improve reliability performance and, in doing so, provide financial benefits to their owners and system operators. The results illustrate how all stakeholders might benefit from a smart grid deployment and control of ES, which can ensure higher reliability and continuity of supply.

This article is organised as follows: Section 2 presents the realistic large MV/LV urban distribution network used for the analysis, detailing the aggregation techniques for reducing the complexity of involved calculations. The reliability assessment methodology is presented in Section 3, while a number of scenarios designed to demonstrate the comparative benefits in network reliability performance by the deployment of different DERs is detailed in Section 4 . The network reliability performance, quantified by the standard set of reliability indices, such as energy not supplied (ENS), frequency and duration of long (LI) and short (SI) interruptions, is presented and discussed in Section 5. Finally, Section 6 lists the main conclusions from the presented work.

\section{MV and LV Distribution Network Design}

To demonstrate the impact of DERs on reliability performance, this research utilises a generic distribution network as well as novel aggregation techniques to accurately model a large MV network. Load may generally be classified as being residential, commercial and industrial at either LV or MV level. After load identification at each network node, generic network models are used to represent the actual distribution systems [18]. Network models emulate typical configurations e.g., enclosed substations and short-length underground cables in cities (due to high load density), and outdoor substations and overhead lines in rural areas. The aggregation methodology is detailed in the next subsection, followed by the selected urban MV network model used for the reliability study, which incorporates the LV network electrical and reliability equivalents.

\subsection{Electrical and Reliability Network Equivalents}

For a comprehensive QS analysis, it is important to build the accurate and flexible network equivalents from generic models of LV and MV distribution networks, and identify the typical associated components. These network equivalents can then be used to formulate corresponding aggregate representations for each load subsector, in which network models are reduced to a single-line equivalent impedance, $Z_{e q}$, therefore reducing system complexity, as well as required computational time and effort. Using the power component (PC) impedances from the LV and MV generic networks from [15], the "bottom-up" aggregation method is applied, starting with the aggregation of individual network components from each LV point of consumption, up to the supplying primary (MV/MV) or secondary (MV/LV) substation [18].

Figure 1 illustrates building of the equivalent network for a LV distribution system, where the equivalent impedance, $Z_{e q, L V}$, represents the overall downstream network impedance. Similarly, the aggregate demand is the sum of all LV customers' loads connected downstream the point of aggregation. The network aggregation methodology presented is based on the work in $[19,20]$, where radial network reduction into single line equivalent impedances was developed. The main purpose was for voltage stability analysis and fast load-flow calculations. This technique is outlined below:

(1) Calculate equivalent impedance at every network load location. This includes multiple customers at the same bus or supply point, and is expressed by Equation (1).

(2) Sum all equivalent impedances calculated in Step 1 to determine the overall network impedance. This is described by Equation (2):

$$
Z_{\text {location }}(i)=\sum_{n_{i}=1}^{N_{i}} Z_{\text {customer }}\left(n_{i}\right)
$$




$$
Z_{e q}=R_{e q}+j X_{e q}=\sum_{i=1}^{I} Z_{\text {location }}(i)
$$

where: $N_{i}$ is the number of customers at load location $i, I$ is the number of load locations, $Z_{\text {customer }}\left(n_{i}\right)$ is the impedance of the customer $n_{i}$ at location $i, Z_{\text {location }}(i)$ is the sum of all customer impedances at location $i$, and $Z_{e q}, R_{e q}$ and $X_{e q}$ are the equivalent impedance, resistance and reactance, respectively.

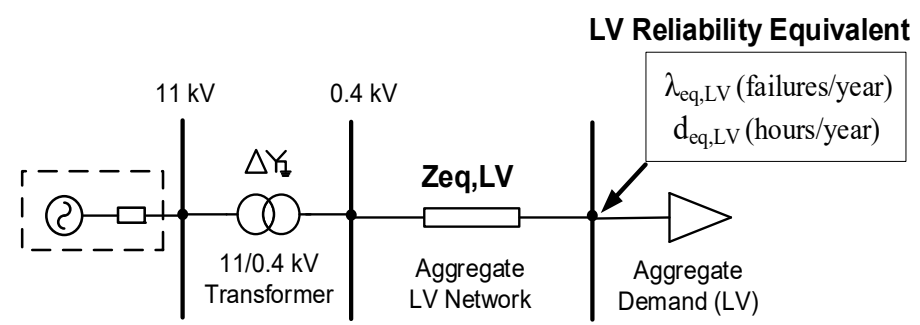

Figure 1. Aggregate representation of LV distribution network, showing the equivalent impedance, aggregate demand of all connected customers and reliability-equivalent model parameters.

The two inputs to the Monte Carlo Simulation (MCS) used for reliability assessment in this paper are the failure rates $(\lambda)$ and mean repair times $(\mathrm{d})$. They describe the reliability-characteristics of all the modelled network PCs [21-23]. However, simple bulk loads are usually used to represent LV and MV parts of power systems due to calculation complexity when using very detailed and/or large models. Reliability indices calculated with this method of aggregation are often inaccurate because failures of PCs in aggregated parts of the network cannot be modelled accurately. Higher accuracy in reliability equivalent models can be achieved if proper (equivalent) failure rates and repair times are allocated to the bus where bulk load is attached. This will then facilitate network reduction through the aggregation and reduction of simulation times. Figure 1 also illustrates the resulting aggregate LV network model after the inclusion of reliability equivalents. The equivalent failure rate, $\lambda_{e q}$, and repair time, $d_{e q}$, of the bus where the aggregate demand is connected are calculated as the sum of the failure rates of all PCs and the average of their repair times, respectively. These are described by Equations (3) and (4). Equivalent parameters, $\lambda_{e q}$ and $d_{e q}$, are derived from the known $\lambda$ and $\mathrm{d}$ values provided by historical data for each relevant PC in the aggregated part of the system. These equivalent parameters consider the reliability performance of every PC (e.g., buses, lines, protection equipment, etc.) downstream the aggregation point and are utilised for ascertaining interruption frequency and duration within the aggregated network [24]:

$$
\begin{gathered}
\lambda_{\text {eq }}=\sum_{i=1}^{M} \lambda_{i} \\
d_{e q}=\frac{1}{M} \cdot \sum_{i=1}^{M} d_{i}
\end{gathered}
$$

where $M$ is the number of PCs, $\lambda_{i}$ and $d_{i}$ are individual PC failure rates and repair times, respectively, and $\lambda_{e q}$ and $d_{e q}$, are the equivalent failure rates and repair times, respectively, for the whole aggregated network represented with the reliability equivalents.

\subsection{Urban MV/LV Distribution Network}

The residential load sector can be categorised into four subsectors, where a different network model can represent each. These models are classified based on the location, size and type of dwellings into four subsectors: highly-urban, urban, suburban and rural generic LV distribution networks. For the urban residential subsector, Figure 2 provides a generic LV distribution model illustrating an underground cable arrangement operated in a radial configuration. A transformer typically rated 500 $\mathrm{kVA}, 11 / 0.4 \mathrm{kV}$ (for UK) is used for this type of networks, supplying a total of 19 load points, U1-U19, 
with a total of 190 single-phase connected customers. The network has four three-phase trunk feeders, which are supplied from the LV busbars of the infeeding 11/0.4 kV secondary substation. The network is characterised by relatively short conductor lengths, to ensure voltage regulation within the specified limits, i.e. $+10 /-6 \%$ in the UK. Detailed data about line parameters are provided in [25].

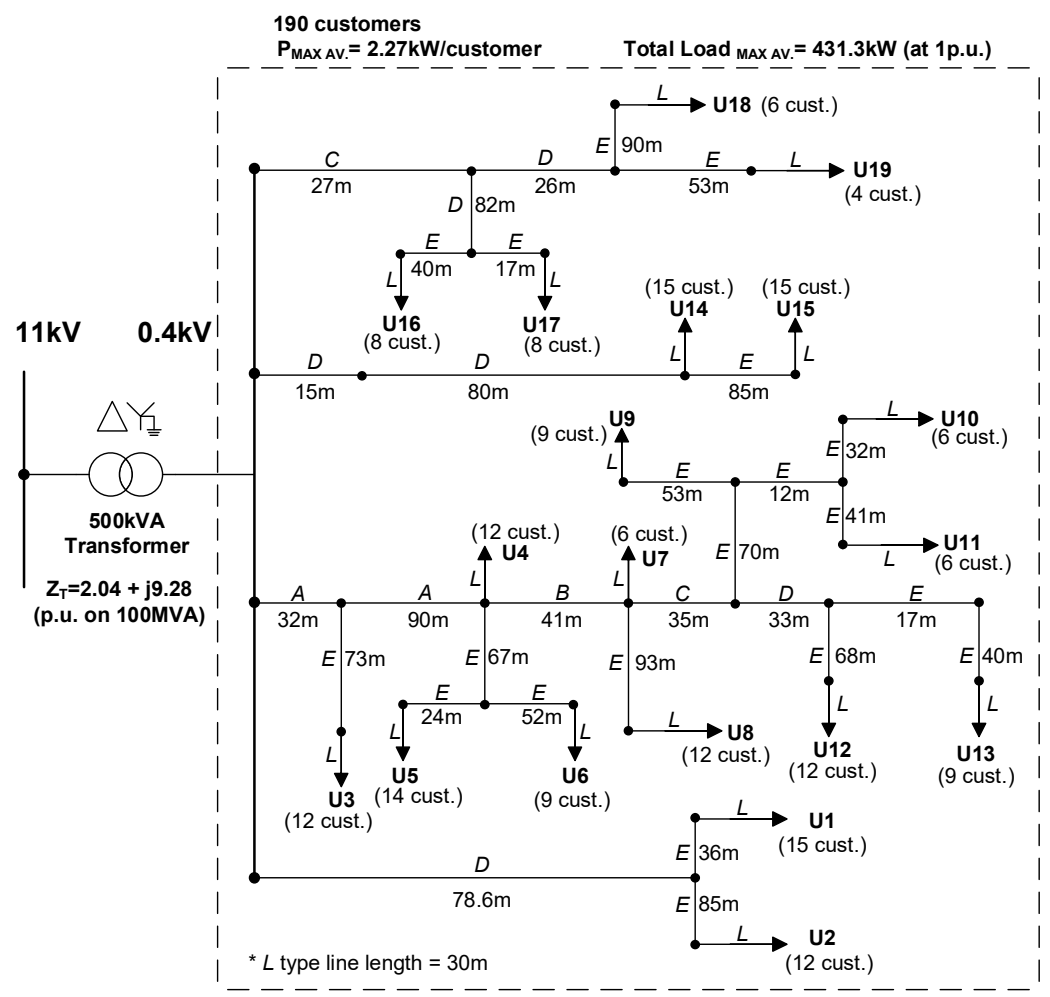

Figure 2. Urban generic LV distribution network model.

The use of the previously described aggregation techniques reduces the complexity and computational times associated with modelling very detailed networks, such as the one shown in Figure 2 [26]. To perform a reliability assessment study on a MV urban distribution network in Figure 3, the LV network from Figure 2 is aggregated and represented by its electrical and reliability equivalents.

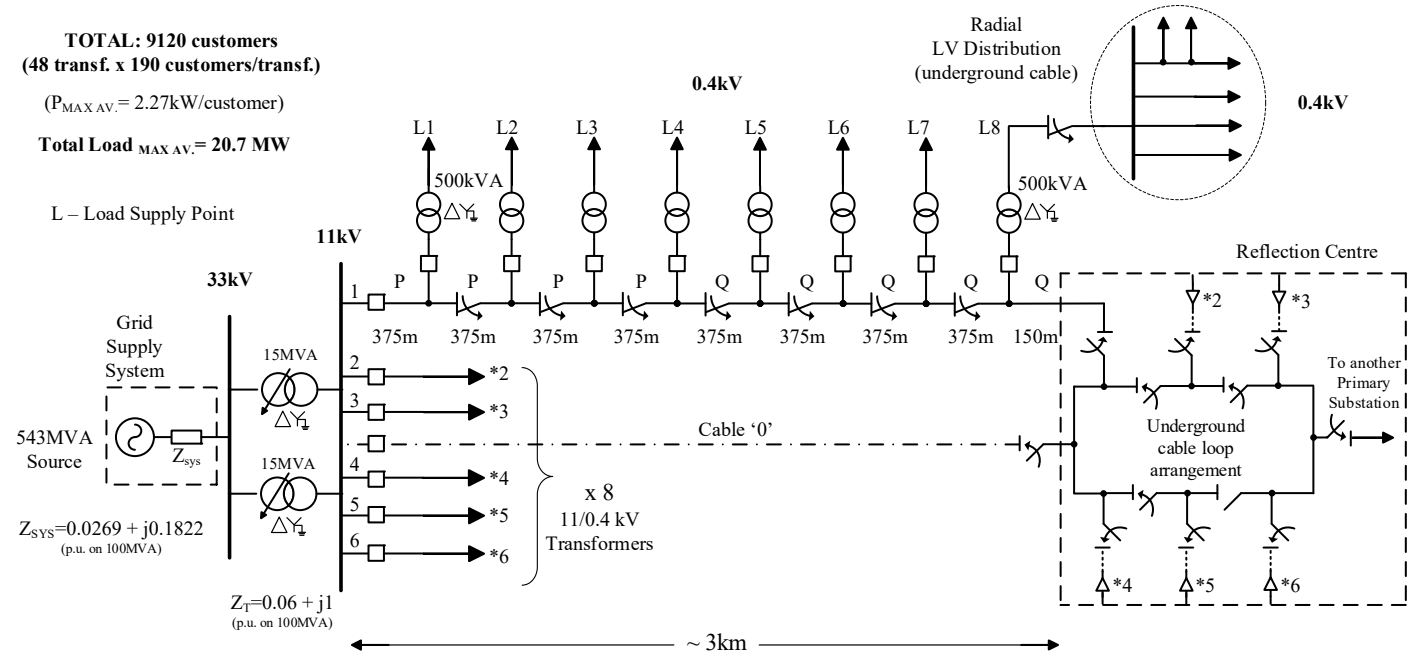

Figure 3. Urban generic MV distribution network model incorporating aggregated LV models from Figure 2 (with corresponding equivalent failure rates and mean repair times). 
Afterwards, each $11 \mathrm{kV}$ load supply point in the MV network can be substituted with the aggregated urban generic LV network (Figure 2), where electrical characteristics, e.g., power flows and voltage profiles, in the MV network, remain unchanged from the detailed network models. A typical $33 \mathrm{kV}$ source strength of $543 \mathrm{MVA}$ is considered for this type of network, which typically supplies a significant percentage of residential loads, here modelled with only residential demand, spanning a radius of approximately $3 \mathrm{~km}$. The network has an underground meshed configuration and operates with six identical trunk feeders, each supplying eight bulk supply points (BSPs), with the total of 48 BSPs. Each of these is supplied by a secondary substation (11/0.4 kV transformer), as in Figure 2, representing the total aggregate demand of 9120 residential customers.

The total load is approximately 3.4 MW during minimum and $21 \mathrm{MW}$ during maximum loading conditions. Although the underground MV networks are typically designed in a meshed configuration, they are normally operated radially, with the support of another supply point, e.g., another similar/same MV primary substation. A closed-loop design that guarantees the supply in case of an $\mathrm{N}-1$ failure is achieved through normally open tie-switches at the end of feeders. This is illustrated in Figure 3 by the cable ' 0 ', i.e., feeder connecting both ends of the network (no load in normal operation).

\section{Reliability Assessment Methodology}

As mentioned, the main reliability parameters used as inputs to the MCS in this paper are failure rates and mean repair times. These are allocated to each PC before system reliability is assessed. It is previously shown that they are not static average values, but vary over time and are therefore more accurately represented using probability density functions (PDFs). Accordingly, the inverse transform method is used in this paper to generate random variates that follow exponential and Rayleigh distributions for failure rates and repair times respectively. This is necessary to convert failure rates and repair times into the two main system states, Time to Fail (TTF) and Time to Repair (TTR), based on various PC operating and failure stages. Equations (5) and (6) provide general formulae of the considered PDFs as input for MCS. Also, PC failure rates are modelled based on a 'bathtub' distribution curve (time-varying failure rate). This is a more realistic approach, because it captures the fact that there is a higher likelihood of failure when the PC has just been installed and when it is near to the end of its service [27]:

$$
\begin{gathered}
T T F_{\text {Exponential }}=\operatorname{inv}\{1-\exp (\lambda t)\} \\
T T R_{\text {Rayleigh }}=\operatorname{inv}\left\{1-\exp \left(-0.5\left(\frac{t}{\sigma}\right)^{2}\right)\right\}
\end{gathered}
$$

Each simulation run is performed on a year-by-year time basis in 40-year cycles (assumed component's lifetime) and halted when convergence is met, or when 1000 years elapse. Whenever a component fails, a power flow calculation is run to evaluate the number and demand of affected loads. The algorithm is implemented using a combination of MatLab code and PSS/E software, automated using Python. The main steps of the MCS approach used in this paper are encapsulated in Figure 5. Previous work has validated that 1000 years is a sufficient simulation time length to maintain the accuracy of the MCS tool employed for reliability assessment [25]. Notably, MCS (using parallel computing for one-year simulation runs) is used to assess reliability of distribution systems in [28]. However, PC failure rates are not varied over their expected lifecycle and the general simulation resolution is relatively lower $(1 \mathrm{~h})$ than that utilised in this study. Furthermore, this MCS method is enhanced by the use of time-varying electricity demand profiles, as opposed to the regularly used peak or maximum demand [4]. These profiles represent residential load models containing demand decomposition into different load types over the $24 \mathrm{~h}$ of the day, and therefore more accurately representing actual values of both active and reactive power demands.

An important feature of the presented analysis is that there is more accurate correlation between the moments when faults occur and the actual load demand interrupted (i.e. not simply the rated 
power). This further improves the overall accuracy especially when calculating energy reliability indices (e.g., ENS index). This MCS procedure also examines the extents to which the probabilities of network outages are affected by the overall system loading and network conditions. A theoretical supply interruption model in Figure 4 is integrated into the analysis for assessing more accurately the moment in time when interruptions to electricity customers are likely to occur. This is implemented by incorporating probability profiles of both SI and LI, giving "time of the day interruption probabilities" over the $24 \mathrm{~h}$ of the day into the MCS algorithm, as presented in Figure 5. The theoretical model [29] is constructed from previously recorded SI and LI statistics by two different European DNOs. These were particularly important for producing the empirical interruption probability models, which are applicable when specific SI/LI statistics are unavailable.

Together with a comprehensive database of PCs from the UK DNOs, this model allows to reproduce more accurately the stochastic characteristics of the network reliability performance. Different sets of DER schemes are then deployed on this base case network, e.g., using ES systems to compensate for the intermittency of PV DGs, as well as tailored DSR schemes, based on the theoretical interruption model. Design and implementation of these smart grid technologies and functionalities, denoted in the further text as "smart interventions", are described in detail in the next section. The replicability and flexibility of this methodology is thoroughly justified in published work by the authors, e.g., in $[2,4,9,24,29,30]$, where the same core technique is applied to different ranges of high-to-low voltage network configurations, load subsectors, and different type and penetration of DERs.

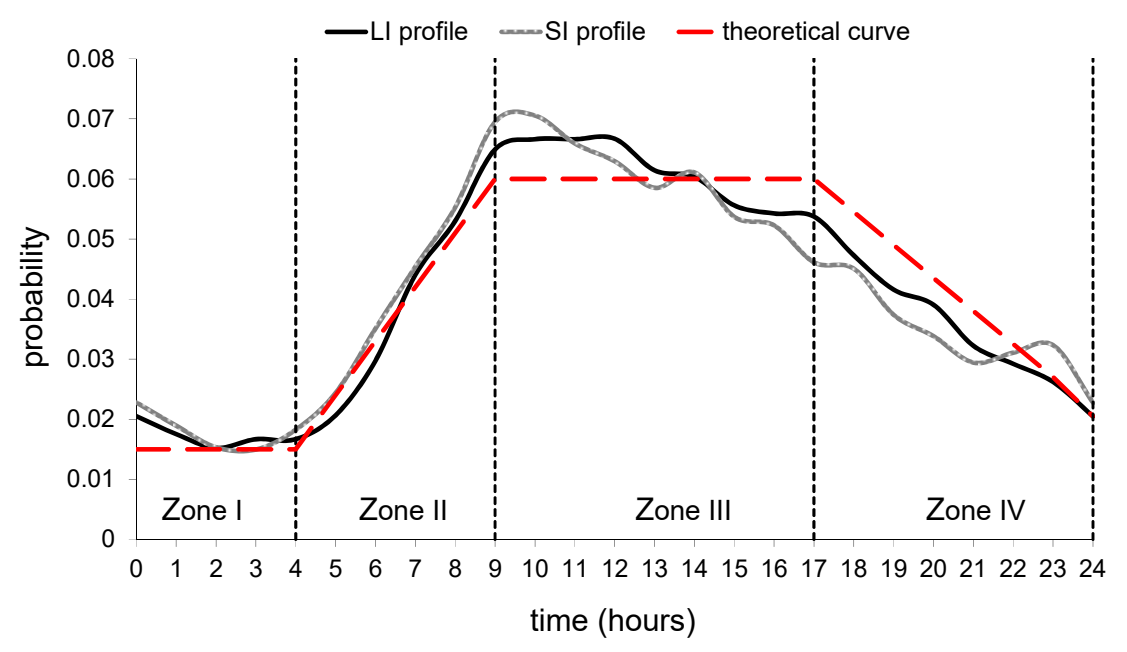

Figure 4. Daily probability of long and short interruptions and their approximation with a theoretical curve. 


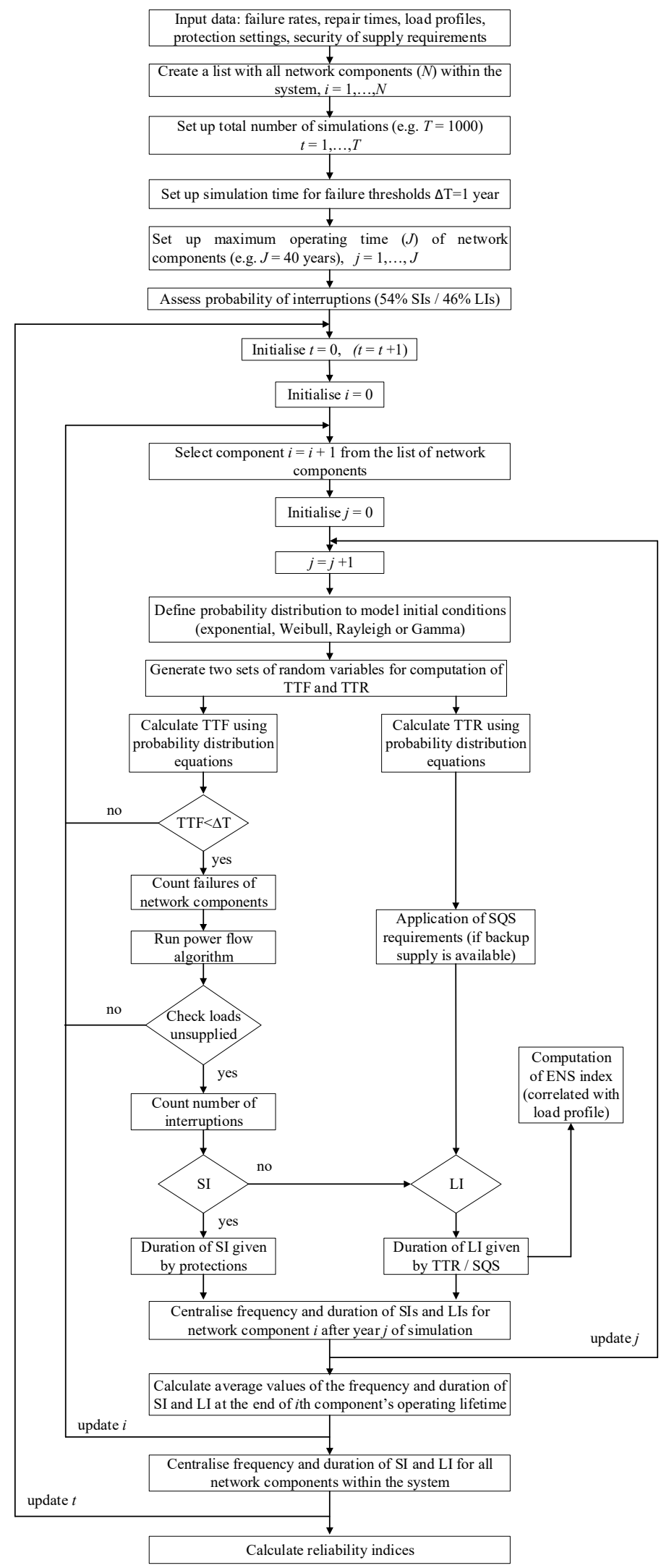

Figure 5. A general algorithm for the MCS-based reliability assessment procedure. 


\section{Network Scenarios Incorporating Smart Interventions}

The test MV distribution system used for analysis (Figure 3) is modelled, scripted and simulated for different network scenarios and functionalities through Python with PSS/E software package. These scenarios are specified in order to evaluate possible improvement or deterioration of network reliability performance, which is quantified through the calculated reliability indices. Accordingly, these scenarios (summarised in Table 1 and detailed in the further text) consider a number of functionalities and aspects of DERs relevant for reliability analysis. The presented analysis provides results not only for the average values of standard reliability indices, but also for their PDFs, to assess more comprehensively the network performance (e.g., risks associated with certain events).

Table 1. Summary of Network Scenarios.

\begin{tabular}{ccc}
\hline ID & Network Scenario & Description \\
\hline SC-1 & Base case & Inclusion of backup capability and SQS regulations \\
SC-2 & DSR & Demand-side response for reliability improvement \\
SC-3 & PV & Uncontrolled MG using the most probable PV power output \\
SC-4 & PV + DSR & Combination of PV and DSR \\
SC-5 & ES & EMS-Controlled MG supplying energy per customer per fault \\
SC-6 & ES + DSR & Application of ES after DSR \\
\hline
\end{tabular}

\subsection{SC-1: Base Case}

The reliability performance of the urban MV network shown in Figure 3 is assessed to establish a base case used as a reference for assessing the benefits of other considered scenarios. This base case network is modelled to include backup capability (through normally open switches at the end of the MV feeders), as well as the implementation of SQS regulation-both of which are standard functionalities of urban MV distribution network operation. The merit of the proposed method is that the relative reliability indices are calculated and compared with each other (and against the base case) to quantify the relative improvement. This ensures that uncertainties in data and system requirements are embedded in all the indices. It also ensures that reasonable confidence can be placed in the relative differences and potential benefits of considered scenarios [21].

\subsection{SC-2: Demand-Side Response (DSR)}

Using the theoretical interruption model presented in Figure 4, a novel DSR scheme is evaluated. During the period with a high fault probability, which is before the evening ramp period, 9:00-17:00 $\mathrm{h}$, "wet load" (including domestic washing machines, dishwashers, etc.) are disconnected. This scenario represents application of 'DSR for reliability improvement', as it should ensure an enhanced reliability performance, as opposed to the more commonly applied DSR techniques, aimed at reducing the evening peak demand [30]. This scenario is not expected to result in significant improvements in reliability, since only a relatively small percentage $(10 \%)$ of the load is disconnected. However, it highlights the potential for reliability improvement and can support further assumptions that more sophisticated DSR schemes can result in significant reliability-performance improvements.

\subsection{SC-3: Uncontrolled PV}

For assessment of reliability benefits from connecting PV MG, the PV power output is modelled considering same output for each residential dwelling. Given that future networks will feature high levels of penetration of DERs [31,32], this scenario illustrates potential benefits of PV integration (with 50\% penetration) and assesses the impact of the spatio-temporal variation of PV systems. Furthermore, the use of a realistic, rather than an ideal PV profile also avoids overestimation of the benefits by accounting for the clouding effect [4], which is detailed in the following sub-subsections. 


\subsubsection{Temporal Variation of Solar Energy Resources}

Most DNOs are reluctant to depend on the PV generation for power delivery capacity, because it does not directly reduce the peak demand. However, it shortens the duration of the peak load, which further benefits loading patterns of PCs [33]. However, unpredictable cloud movements affect the power fluctuations of PV installations and cause voltage fluctuations that often require implementing network controls, or even changing settings of associated protection systems [34,35]. The 'randomness' of input solar irradiance is modelled using the raw measured hourly data from field recordings on a 110-kilowatt peak $(\mathrm{kWp})$ rooftop PV system. Figure 6a shows the measured distribution of PV output power values for each hour of a 'typical' summer day.

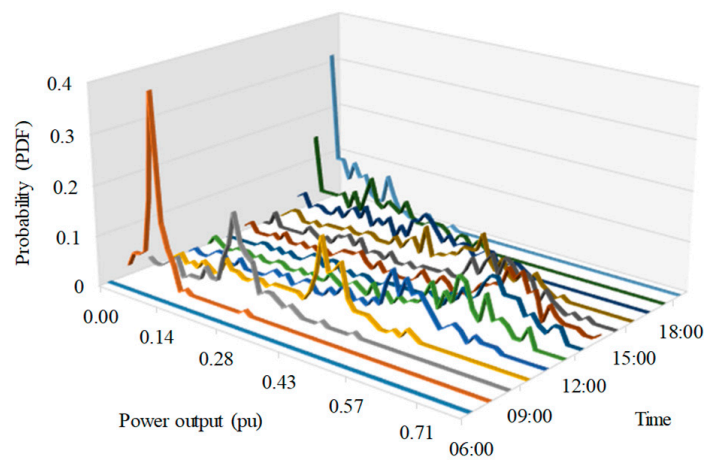

(a)

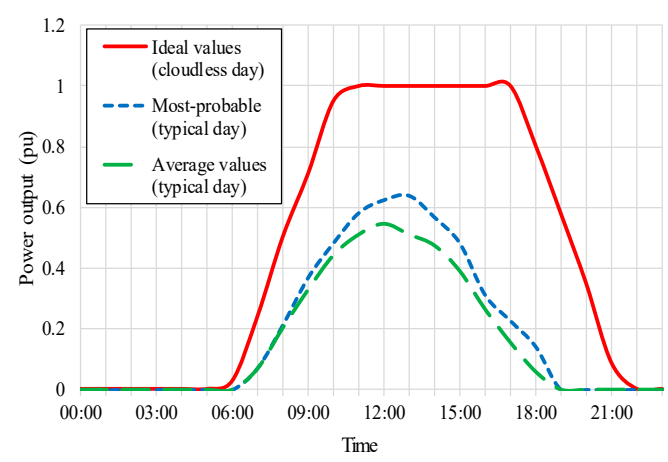

(b)

Figure 6. PV power output modelling: (a) Probabilistic variations of hourly output power; (b) Temporal power output variations for different power output models.

As expected, the probability of low PV output values is high during morning and evening hours, e.g., 0.07 p.u. is the most probable PV power output ( 0.36 probability) at 07:00 h. The use of the most probable values enables modelling of the hourly PV power output for a 'typical' (realistic) day, which is used in the proposed analysis. This is more accurate than using statistical averages, as it accounts for realistic changes in the solar irradiation patterns. The analysis is repeated for cloudless conditions (which are rarely occurring for the whole day), herein termed 'ideal'. Both power output profiles for the realistic and ideal PV generation over a day are compared in Figure 6b, illustrating not only the associated energy loss, but also indicating possible variations in calculated reliability indicators when either PV profile is used for the analysis. For the analysed PV penetration level of $50 \%$, modelled as a uniform distribution of equal-size individual customer PV installations [32], the obtained results quantify the possible overestimation of reliability-based benefits when the 'clouding' effect in PV outputs is not accounted for. Figure $6 \mathrm{~b}$ also shows the average values for hourly PV, output exemplifying the potential underestimation of reliability indices if average values are used.

\subsubsection{Impact of Clouding Effect on Reliability Performance}

Through comparative analysis, reliability benefits of PV integration into the test MV network are assessed using both the realistic PV output profile and the ideal summer maximum one, to represent the range of variation. The results in Table 2 indicate that when a realistic PV profile is used, the reduction in ENS from a base case (i.e., no PV integration) is $16.7 \%$, while it is $38.9 \%$ when the ideal PV output profile is used. This represents a possible overestimation of the benefits of PV by $22.2 \%$, when the variability of input solar irradiance and unpredictable cloud movements is neglected. It means that the projected increase in reliability from the use of ideal PV generation is more than double the expected increase from a realistic PV generation. Similarly, there is an overestimation of the benefits of $\mathrm{PV}$, by at least $22 \%$, for each of the following indices: the system average interruption duration index (SAIDI), average duration of interruptions to only affected customers (CAIDI), and the average 
customer curtailment index (ACCI) [21], which measures ENS for only the customers affected by supply interruptions.

Table 2. Impact of clouding effect on reliability indices.

\begin{tabular}{ccccccc}
\hline Index & Base case & PV & $*$ & Ideal PV & $*$ & Clouding Effect \\
\hline ENS (kWh/cust./y) & 146.37 & 121.90 & $16.7 \%$ & 89.42 & $38.9 \%$ & $22.2 \%$ \\
ACCI (kWh/cust. int.) & 1090.41 & 909.75 & $16.6 \%$ & 664.17 & $39.1 \%$ & $22.5 \%$ \\
SAIDI (hours/cust./y) & 0.550 & 0.453 & $17.7 \%$ & 0.332 & $39.6 \%$ & $22.0 \%$ \\
CAIDI (hours/cust. int.) & 3.678 & 3.043 & $17.3 \%$ & 2.228 & $39.4 \%$ & $22.2 \%$ \\
\hline
\end{tabular}

${ }^{*}$ Reduction from Base Case; cust. = customer; $\mathrm{y}=$ year; int. = interrupted.

However, average sustained-interruption frequency indices for the system (SAIFI) and customers (CAIFI), as well as for momentary interruptions (MAIFI), are not presented in Table 2, as they indicate no over-estimation $(0 \%)$. This is because the major causes of interruptions are system faults and PC failures, which do not affect the solar irradiance and associated PV energy availability, and therefore do not affect interruption frequency indices. PV alleviates effects of LI by providing energy thereby only lessening their duration, but not frequency, as perceived by the customer.

The probability distributions of reliability indices offer more details about the network performance. Figure 7 illustrates the impact of clouding on ENS when PDFs are compared. It shows a reduction in the long tail of the PDF when either 'PV' (most probable) or 'Ideal PV' is integrated into the network. This represents a reduction in the maximum value of the expected range of interruptions, and is a major benefit as the probabilities of lower ENS values increase. There exists a clear overestimation, due to different PV operating conditions, as the maximum ENS values range from 1700 to $2100 \mathrm{kWh} /$ customer/year.

Given that these results are reliant on measured data from PV installations to deduce the most probable PV generation profile, there is a strong correlation between the variation of realistic PV power output and its geographical (or meteorological) location [36], as well as its accuracy and resolution. Higher incidence of unpredictable cloud-movements would lower the overall energy availability, thereby lowering the PV's value in increasing reliability performance. Furthermore, the variability of wind (both stand-alone and in hybrid PV-wind systems) would result in similarly skewed reliability performance results, when the modelling accuracy is the same as in this study.

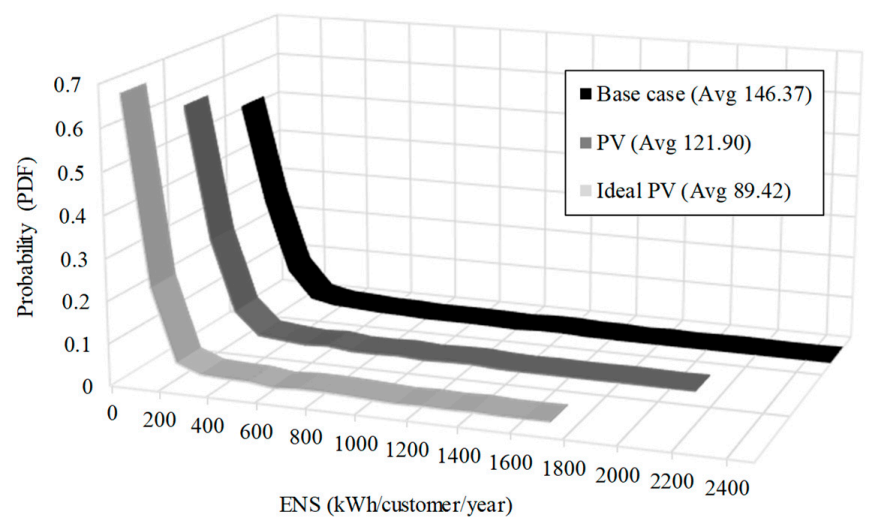

Figure 7. Impact of PV integration on network ENS to customers.

\subsubsection{SC-4: PV+DSR}

This scenario models a coordinated application of PV (with most probable outputs) and DSR strategies from SC-2 to quantify possible additional benefits for both system and customer reliability. The additional effect of preventive DSR actions on top of the variable PV generation is expected to reduce uncertainty resultant from unpredictable PV power outputs and provide an improved level of supply continuity to customers. 


\subsection{SC-5: Energy Storage (ES)}

While many control techniques for ES focus on peak shaving applications and energy cost reduction [5,37], this paper analyses specific smart application of ES, which is designed to improve reliability performance by providing a backup capacity per customer, per fault, with the intention of reducing the ENS and duration of sustained interruptions. The selected backup capacity is $3.67 \mathrm{kWh}$, based on the energy networks association (ENA) G83 engineering recommendation in [38] for peak power that can be provided by a small-scale single-phase rooftop PV. ES operation is controlled by an EMS to provide seamless power switching capabilities and continuous supply to the end-customers. The energy is stored from PV MG operating in islanded mode and is expected to result in a better reliability performance than the uncontrolled PV (SC-3). While similar scenarios in previous work $[4,30]$ modelled ES systems with constant state of charge (SOC) characteristics, this paper uses a realistic SOC-level variation and thus provides more realistic results. The SOC is modelled based on solar irradiation, load demand, and electricity tariff during grid supply conditions. Therefore, the EMS is modelled to capture more accurately the SOC and the available ES output power. The temporal variations of these quantities then determine SOC variations. Accordingly, this model utilises PV generation, residential load demand, and electricity tariff data. To prevent overheating and ensure long battery lifetime, SOC limits are set to $40 \%$ and $100 \%$ as previously utilised in [39,40]. The ES SOC capacity is modelled following equation (7) [41] which fully considers both charging and discharging efficiencies of the ES system; the latter being higher as fully detailed in [42,43]:

$$
\operatorname{SOC}(t)=\operatorname{SOC}(t-1)+\eta_{c h} \cdot\left(P_{c h}(t) \Delta t\right)-\frac{P_{d i s}(t) \Delta t}{\eta_{d i s}}
$$

where $S O C(t)=S O C$ of the battery at time $t(\mathrm{kWh}), P_{c h}$ and $P_{d i s}$ are the electrical power input and output respectively at time $t$, while $\eta_{c h}$ and $\eta_{d i s}$ are charging and discharging efficiencies respectively.

\subsubsection{ES System Configuration}

The EMS configuration shown in Figure 8 is based on a real microgrid system tested in the Smart Grid Laboratory at the University of Bath, UK. The designed 12-panel PV system has rated peak power of $3.67 \mathrm{~kW}$ and voltage of $444 \mathrm{~V}$ [44]. A $4 \mathrm{~kW}$ DC/DC buck converter steps down the voltage to a $24 \mathrm{~V}$ rated battery bank (made by series-parallel connection of four $12 \mathrm{~V}$ batteries), which can supply maximum current of $200 \mathrm{~A}$ (corresponding to maximum power of $4.8 \mathrm{~kW}$ ). The converter, battery bank and $10 \mathrm{kVA}$ inverter are linked with a common coupling $24 \mathrm{~V}$ DC bus. The inverter is also connected to the DC bus and allows for a bi-directional energy exchange (and energy trading) via one-phase of a three-phase mains supply [39]. The EMS controls power output from PV either directly, or through the ES, since a grid-connected mode is not designed for ES-charging in this study.

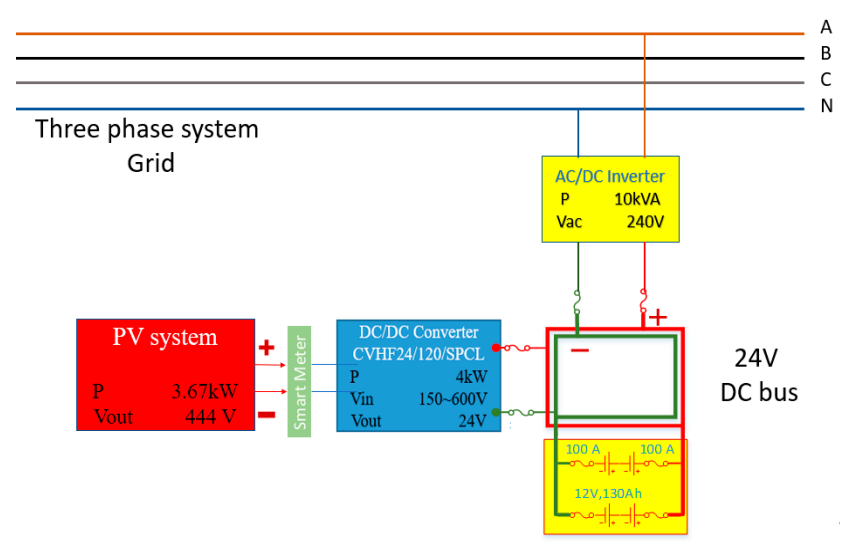

Figure 8. Single-phase EMS configuration. 


\subsubsection{ES System Operation}

Figure 9a presents the SOC for the ES system. For a typical day, system operation is suitably described as follows: the initial SOC may be assumed $40 \%$ at 00:30 h. It essentially remains at this level until 05:00, when PV starts to generate and charge the ES by 07:00. Between 07:00 and 11:00, ES remains at approximately 100\% SOC. It then discharges due to increased demand (between 11:00-15:00) and charges up again during a relatively low tariff period (15:00-18:30). Evening peak demand is met, partly, by discharging the ES system up to 22:00 where the EMS takes advantage of the low demand and low rate late-night tariffs to charge the ES. Figure $9 b$ shows the power output: positive values indicate discharging while negative values correspond to charging. System operation assumptions include no charge-discharge losses, no occurrence of unexpected failures (no failure rate), and ES apparatus is not damaged by large upstream network faults [9].

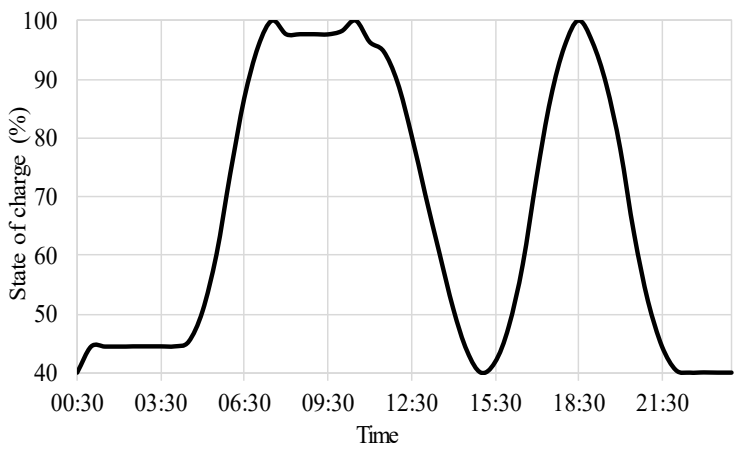

(a)

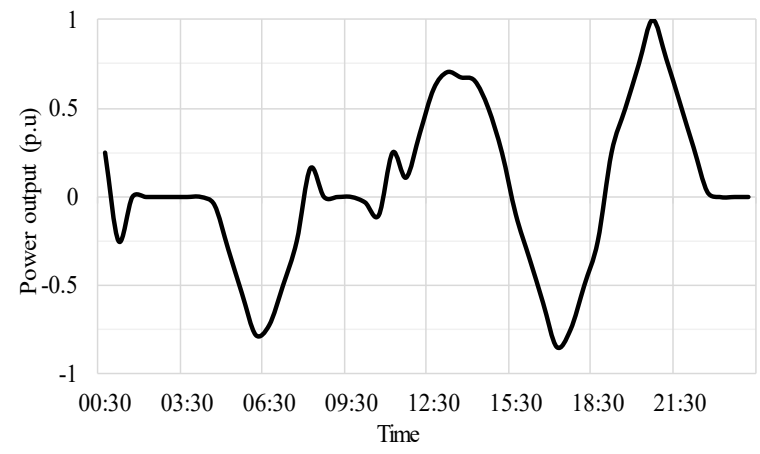

(b)

Figure 9. Battery SOC and power output for a typical summer day: (a) State of charge; (b) Power output.

\subsubsection{SC-6: ES + DSR}

In this scenario, ES provides backup capabilities by providing supply to individual customers in the event of a network outage. The EMS-controlled ES is deployed as a well-designed corrective action on top of a preventive DSR application (SC-2). In case of an upstream fault (whose effect is reduced due to DSR action), the EMS will provide additional power, available from either PV system, or ES, or both, in order to supply all loads and thus significantly reduce the duration of supply interruptions, or even completely prevent them. Therefore, this combination of several smart grid technologies for a localised and smart energy management ("smart interventions") is expected to result in the highest benefits for system and customer reliability performance.

\section{Reliability Performance Assessment}

The average values of system reliability indices are presented in this section. These include indices that measure the frequency of SI (MAIFI) and LI (SAIFI and CAIFI), the duration of LI (SAIDI and CAIDI) and energy not supplied (ENS and ACCI). Additionally, PDFs and cumulative density functions (CDFs) of these indices are also presented, to illustrate the stochastic variability as well as the potential benefits that may not be immediately obvious. Notably, the application of DSR on its own in SC-2 (Table 1) does not offer significant benefits, as only the wet loads are disconnected during a span of about $10 \mathrm{~h}$ in the day. This results in a 7.7\% reduction of ENS from the base case, but offers no significant improvements in other indices, related to frequency and duration of outages. This is why SC-2 is not assessed individually in the following reliability performance analyses. Major benefits from this scenario are realised mainly through the aggregation with other smart grid technologies, such as PV and ES, which are related to scenarios SC-4 and SC-6, respectively. 


\subsection{Impact on Frequency of Interruptions}

Table 3 presents the results for the network reliability performance by considered smart interventions, where their benefits are quantified by the reductions of index values relative to the base case. The results show that the controllability of MG using ES is important for reduction of SAIFI-71.5\% and 75\% reduction in the ES and ES + DSR scenarios, respectively. Furthermore, the results illustrate that even the interruption frequency when assessed only for those customers affected by interruptions (CAIFI) is reduced significantly, i.e. CAIFI is $19.4 \%$ and $22.6 \%$ lower than for the base case, when ES and ES + DSR are deployed, respectively. The enhancement offered by ES is further highlighted when the number of customers affected is assessed before/after the application of ES. It can be seen that the average number of load supply points affected by interruptions reduces from nearly 7 in the base case, to roughly 2 when either ES scenario is deployed. However, Table 3 highlights the advantages of PV-shown by no change in MAIFI, which is better than the $4.4 \%$ and $3.7 \%$ increases when ES scenarios are deployed, as this can not only negatively affect the power quality, but also require changes to the settings of the main substation protection schemes [45]. This increase in MAIFI index is due to the use of backup ES, which can alleviate faults in the upstream network, either by total removal of the outage or by converting LI into SI. However, this is not an evident result from the PV scenarios (SC-3 and SC-4), as no intelligent control is designed.

Table 3. Frequency of long and short interruptions.

\begin{tabular}{cccccccc}
\hline Scenario & $\begin{array}{c}\text { MAIFI } \\
\text { (Ints/c/y) }\end{array}$ & $*$ & $\begin{array}{c}\text { SAIFI } \\
\text { (Ints/c/y) }\end{array}$ & $*$ & $\begin{array}{c}\text { CAIFI } \\
\text { (Ints/Affected } \\
\text { Customer) }\end{array}$ & $*$ & $\begin{array}{c}\text { Load Points } \\
\text { Affected (avg) }\end{array}$ \\
\hline Base case & 0.208 & - & 0.157 & - & 0.720 & - & 6.644 \\
PV & 0.208 & $0 \%$ & 0.157 & $0 \%$ & 0.720 & $0 \%$ & 6.644 \\
PV + & 0.208 & $0 \%$ & 0.157 & $0 \%$ & 0.720 & $0 \%$ & 6.644 \\
DSR & 0.218 & $-4.4 \%$ & 0.045 & $71.5 \%$ & 0.581 & $19.4 \%$ & 1.841 \\
ES & 0.216 & $-3.7 \%$ & 0.039 & $75.0 \%$ & 0.557 & $22.6 \%$ & 7.643 \\
ES + DSR & \multicolumn{7}{c}{ * Reduction from Base Case; ints = interruptions; c/y = customer/year. }
\end{tabular}

\subsubsection{System-Oriented Frequency of Interruption Indices}

Figure 10 provides important additional information to the average values in Table 3, showing the PDFs of SAIFI and MAIFI indices, respectively. Figure 10a illustrates that ES + DSR significantly increases the probability of 'zero' LI, while PV + DSR offers almost no improvement to the base case. This can be attributed to the fact that in SC- 6 , the energy stored is utilised as a corrective action when network faults occur, yet no control measures are implemented in SC-4. The PDF for the frequency of SI is plotted in Figure 10b, showing that the little to no degradation from the base case MAIFI. Therefore, this methodology is capable of accounting for both SI and LI; producing accurate results that correspond to the estimated reliability performance. As expected, both scenarios do not significantly affect average MAIFI values as discussed in Section 4.3.2. Furthermore, both graphs in Figure 10 show a spiky increase in the probability value at 1 interruption/customer/year, which is due to system faults affecting all supplied customers, e.g., due to a fault at the primary substation (when both supplying $33 / 11 \mathrm{kV}$ transformers fail) with no alternative (backup) supply to customers. 


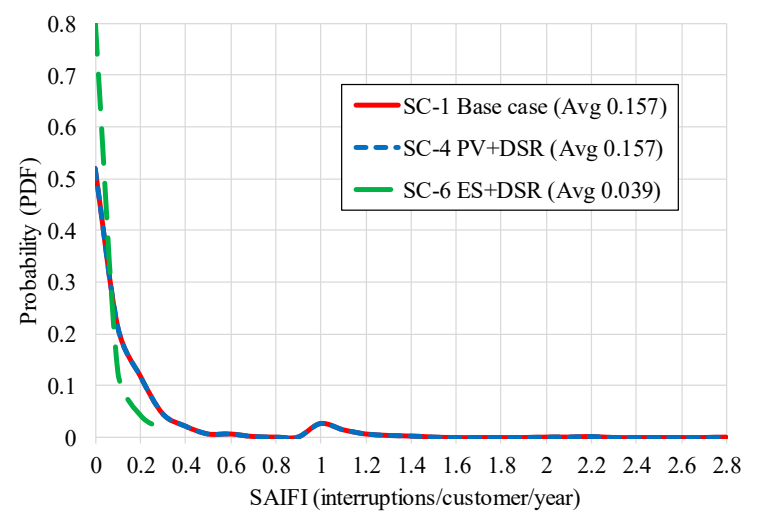

(a)

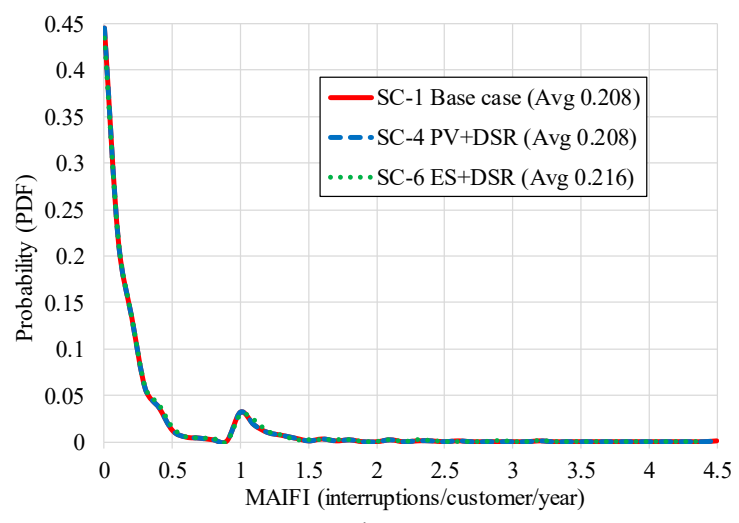

(b)

Figure 10. Probability distribution of the frequency of (a) sustained and (b) momentary interruptions.

\subsubsection{Customer-Oriented Frequency of Interruption Indices}

CAIFI is particularly useful in recognising chronological trends in the reliability of a distribution network, highlighting the years when not all supplied customers are affected by interruptions, and many experience continuity of supply [21]. Table 4 further analyses this index by focusing on the probability of supply interruptions to a particular number of load points. It shows that while PV + DSR offers no discernible improvement to the number of load points affected by interruptions, ES + DSR increases the probability of not having any load point affected by interruptions by almost $60 \%$. Furthermore, using ES + DSR increases the probability of having only one load point affected by $55 \%$. As a direct consequence of those increments, Table 4 also shows that the probability of LI affecting anywhere between 2 and 47 load points is nearly halved in this scenario.

Table 4. Probability of the number of load points affected by supply interruptions.

\begin{tabular}{cccccc}
\hline \multirow{2}{*}{$\begin{array}{c}\text { Number of Load } \\
\text { Points Affected }\end{array}$} & SC-1: Base Case & \multicolumn{2}{c}{ SC-4: PV + DSR } & \multicolumn{2}{c}{ SC-6: ES + DSR } \\
\cline { 2 - 6 } & Probability & Probability & $*$ & Probability & $*$ \\
\hline 0 & 0.320 & 0.320 & $0 \%$ & 0.508 & $-58.8 \%$ \\
1 & 0.147 & 0.147 & $0 \%$ & 0.228 & $-55.1 \%$ \\
$2-47$ & 0.471 & 0.471 & $0 \%$ & 0.261 & $44.6 \%$ \\
48 & 0.062 & 0.062 & $0 \%$ & 0.003 & $95.2 \%$ \\
\hline
\end{tabular}

* Reduction from Base Case.

Most importantly, the results illustrate a significant improvement for SC-6, as this intervention can lower by $95 \%$ the probability of having all load points (48) affected by interruptions. The results show that in 1000 years, all load points are affected by interruptions only 3 times when ES + DSR is used, as compared to 62 times when no smart interventions are deployed. The results in Table 4 are further detailed in Figure 11. It can be seen that ES + DSR significantly increases the probability of having a lower number of load points affected by interruptions, while substantially decreasing the probability of having a greater number of load points affected. In some cases, the reduction is so great that it completely negates number of interruptions experienced by that number of load points. This is exemplified by the zero probabilities for the number of interrupted load points of 17-24, 26, 30, 32 and 33, where the use of ES + DSR results in no interruptions for these number of load points. 


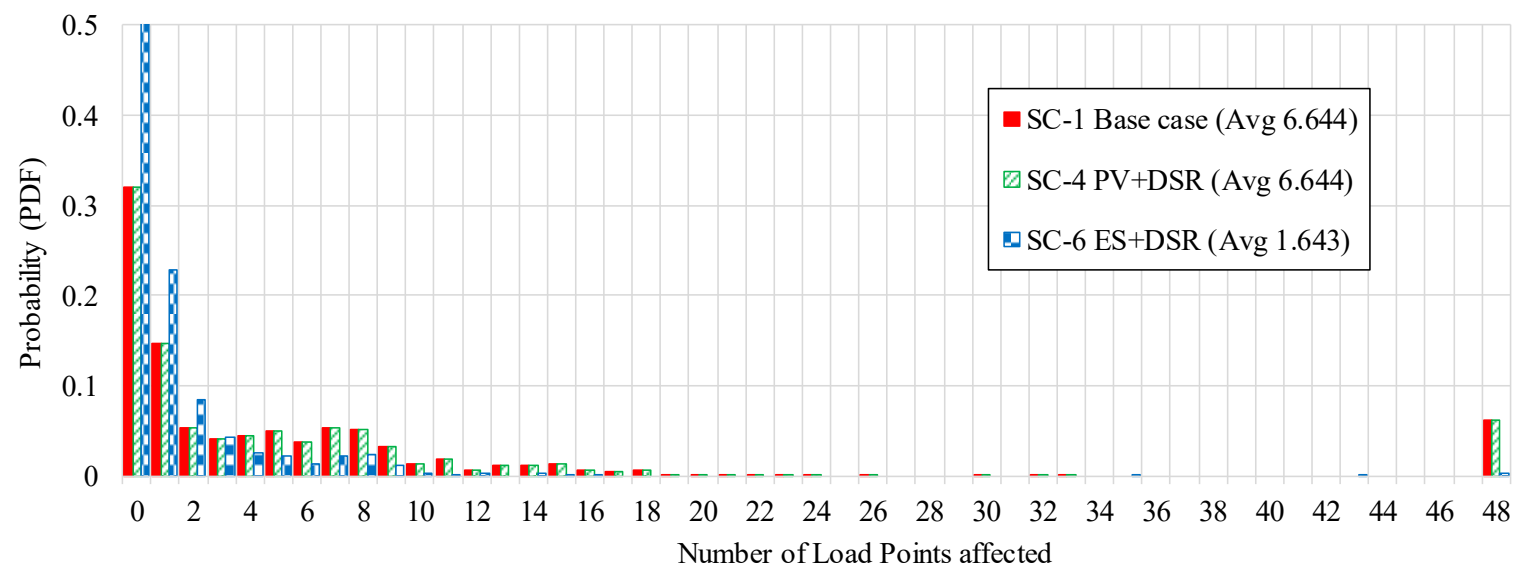

Figure 11. Probabilities of the number of load points affected by supply interruptions.

The scatter plot in Figure 12 further demonstrates the capability of ES + DSR to 'confine' the effect of supply interruptions to much fewer load points than in the base case scenario and also lower the total number of affected customers. The equivalent CAIFI results presented in Figure 13 further confirm the benefits of the ES + DSR scenario.

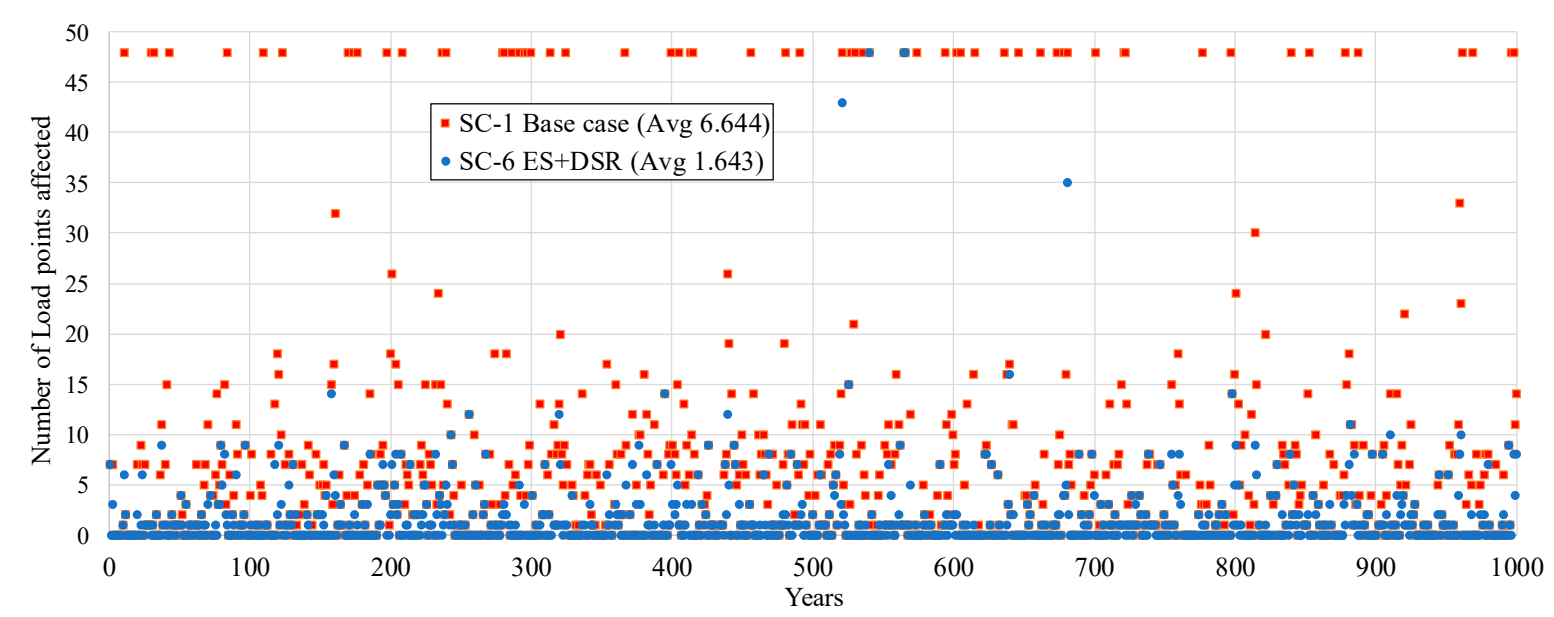

Figure 12. Number of load points affected by supply interruptions.

Notably, all considered scenarios (SC-1, SC-4 and SC-6) do not have any values occurring within the range $0-1$, because CAIFI is only calculated for customers affected by interruptions, implying that the individual average CAIFI values can only be less than one if they are zero (when no load point is affected by interruption)-i.e. no values between $0-1$. From a base case CAIFI average value of 0.72 interruptions per customer interrupted, SC-4 (PV + DSR) does not alter this average value. However, this CAIFI value is further reduced by $22.6 \%$ to 0.557 when SC-6 (ES + DSR) is deployed. This illustrates the substantial improvement offered by coordinated ES + DSR technologies in terms of frequency of interruptions of affected customers.

Figure 13 also proves that while SC-6 (ES + DSR) can benefit the system reliability performance by increasing the probability of a lower number of interruptions for affected customers, it simultaneously increases the largest plausible number of interruptions that can be experienced at a single load point: from 2.8 in the base case to 4 . This effect is best explained by the fact that the EMS-controlled ES is deployed as a corrective action when faults occur. While in most cases it can completely ensure supply continuity by alleviating the effects of upstream network faults, in the cases where it only lowers the interruption duration, the net effect is to have fewer customers affected for the same number of interruptions, therefore resulting in a higher ratio of interruptions per affected customer, i.e., higher CAIFI. 


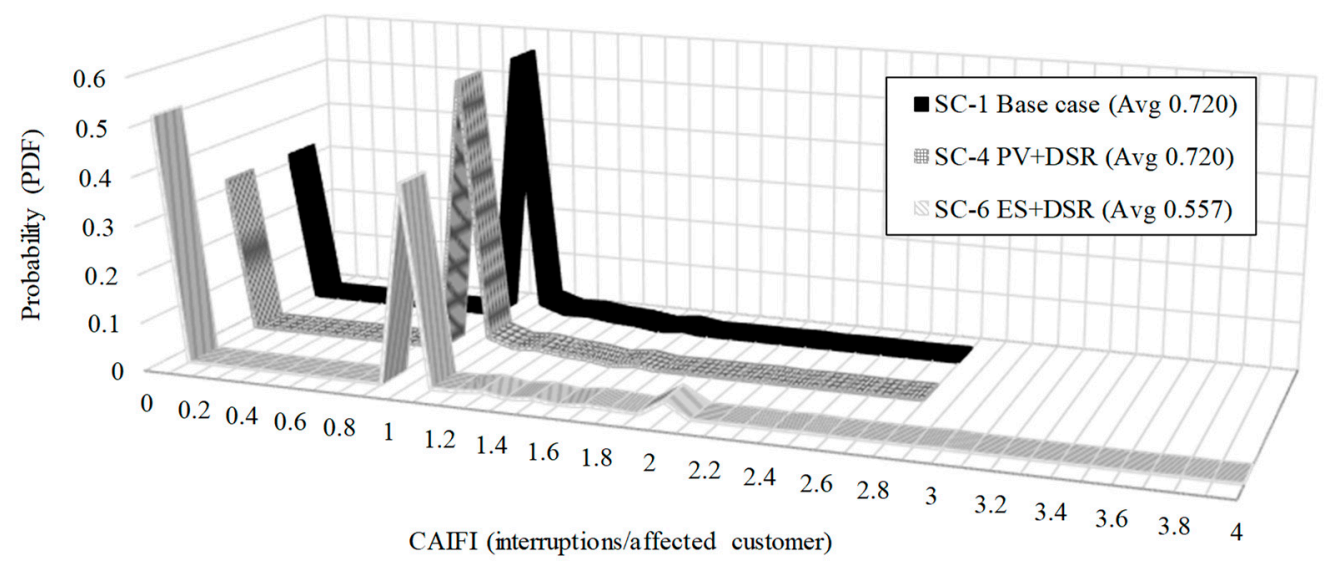

Figure 13. Comparison of PDFs for the CAIFI index.

This is further discussed in Section 5.3, where results for the ACCI index are presented. In summary, ES + DSR reduces the number of customers affected significantly more than it reduces the total number of customer interruptions, thus resulting in a highly plausible CAIFI. In addition, Figure 13 illustrates a 'peak' around the value of CAIFI $=1$ due to the coincidence of faults and number of affected customers, especially when system faults affect large parts of the network and lead directly to interruptions of loads, as highlighted in Figure 10.

\subsection{Impact on Duration of Sustained Interruptions}

Deployment of PV+DSR in SC-4 results in a reduction of interruption duration times by $26 \%$ (Table 5). Moreover, a more controlled application of this RER using an EMS-controlled ES combined with DSR (SC-6) results in nearly a halving (48.7\%) of the expected average interruption duration times. This is a significant result for DNOs, as they can use such a proposed technique as a planning tool to take advantage of this improvement of reliability performance in both technical and commercial terms i.e. in avoiding regulator-imposed penalties for non-satisfactory performance. However, these results suggest that coordinated operation of ES increases CAIDI, which is shown by a $67.8 \%$ and $69.7 \%$ increase of CAIDI for SC-5 and SC-6, respectively. However, this 'increase' is due to the fact that CAIDI is directly calculated from the ratio of SAIDI to SAIFI, and thus it is dominated by the greater reduction of SAIFI (71.5\%) than the reduction of SAIDI (43.7\%).

Table 5. Duration of sustained interruptions: system and customer-based indices.

\begin{tabular}{ccccccc}
\hline ID & Scenario & $\begin{array}{c}\text { SAIDI } \\
\text { (hours/cust./y) }\end{array}$ & $*$ & $\begin{array}{c}\text { CAIDI } \\
\text { (hours/cust. int.) }\end{array}$ & $*$ & $\begin{array}{c}\text { Average } \\
\text { Duration of LI } \\
\text { (hours) }\end{array}$ \\
\hline SC-1 & Base case & 0.550 & - & 3.678 & - & 3.507 \\
SC-3 & PV & 0.453 & $17.7 \%$ & 3.043 & $17.3 \%$ & 2.888 \\
SC-4 & PV + DSR & 0.407 & $26.0 \%$ & 2.751 & $25.2 \%$ & 2.595 \\
SC-5 & ES & 0.310 & $43.7 \%$ & 6.172 & $-67.8 \%$ & 1.975 \\
SC-6 & ES + DSR & 0.282 & $48.7 \%$ & 6.243 & $-69.7 \%$ & 1.800 \\
\hline
\end{tabular}

* Reduction from Base Case; cust. = customer; $\mathrm{y}=$ year; int. = interruption.

\subsubsection{System-Oriented Duration of Interruption Indices}

The resulting PDFs for SAIDI index in Figure 14 reveals that ES (SC-5) and ES + DSR (SC-6) scenarios have reduced tails, which translates to lower probabilities of long-duration interruptions, as compared to the base case. Both ES scenarios also increase the probabilities of the short-duration interruptions, increasing their probability from only 0.33 in the base case, to 0.54 and 0.56 in SC-5 and SC-6, respectively. Similarly, PV scenarios (SC-3 and SC-4) reduce the duration of the longest interruptions, as well as marginally increase the probability of short-duration interruptions. This result 
is due to ES being applied as a corrective action after the faults, while PV is applied as a preventive measure. Therefore, ES does not offer as much reduction in the duration of the longest interruptions as PV, whose deployment offers available energy to alleviate effects from upstream network faults, even without intelligent control from EMS. This might place the use of standalone PV systems, without ES, as a more economical option. However, when pros and cons of either technology are weighed, the impact of ES is controllable and therefore far more predictable than the uncontrolled PV. The larger benefits from ES + DSR are exemplified by nearly a halving of the maximum plausible duration of individual interruptions, which reduces from over $300 \mathrm{~h}$ in the base case, to only $156 \mathrm{~h}$ in the SC-6 scenario.

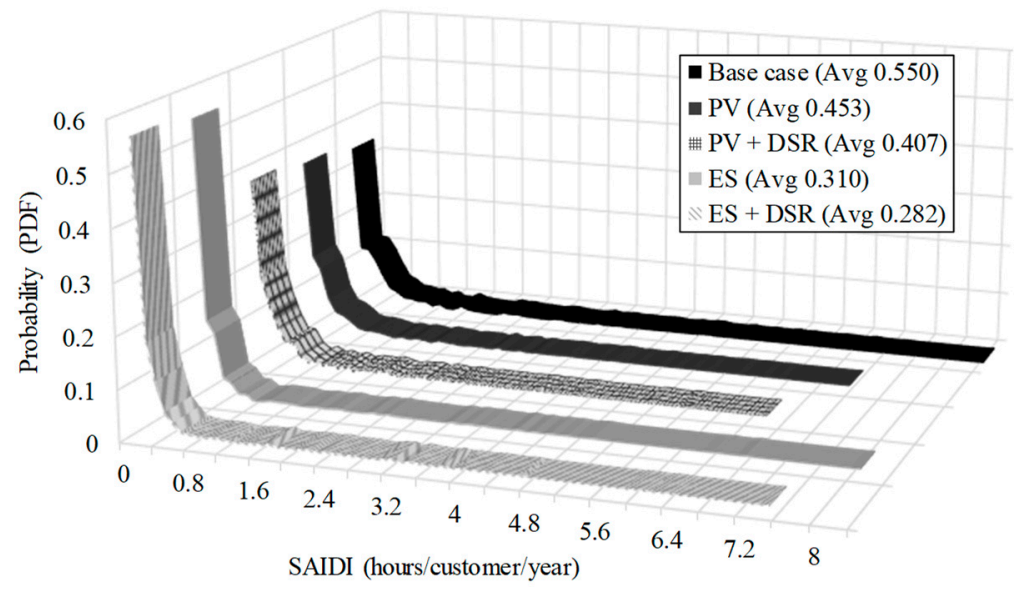

Figure 14. Comparison of the PDFs for the SAIDI index.

Finally, Figure 15 shows the resulting CDFs for the duration of interruptions from three scenarios: SC-1, SC-4 and SC-6. It illustrates that the probability of up to 1-h supply interruptions increases from $54 \%$ in the base case to $81 \%$ when SC- 4 is deployed, and finally to $91 \%$ for SC-6. The results in Figure 15 actually quantify the risk of paying compensation to customers experiencing interruptions longer than the specified duration threshold (as in the UK SQS, [6]). This can be associated with the considered scenarios and smart grid functionalities, which then can be used to evaluate potential cost-benefit effects at both the planning and operational stages. Furthermore, it can be analysed in terms of not only QS improvements to customers, i.e. through the reductions of average duration of long interruptions, but also in terms of the higher probabilities of shorter-duration interruptions.

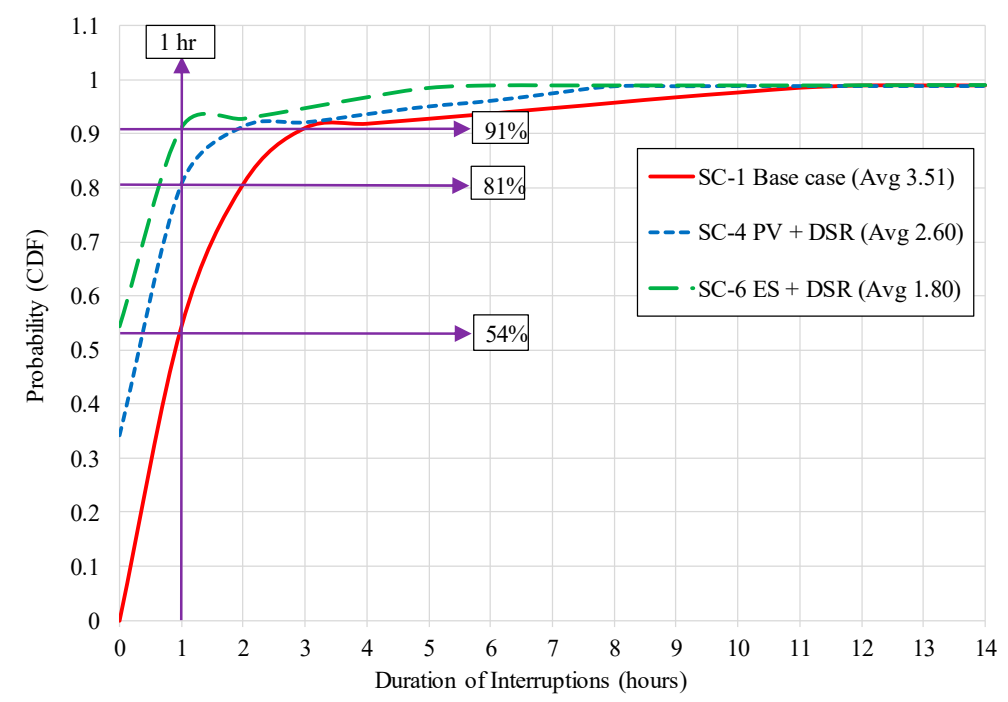

Figure 15. Comparison of CDFs illustrating the impact on the duration of interruptions. 


\subsubsection{Customer-Oriented Duration of Interruption Indices}

Figure 16 presents the PDF plots of the CAIDI index, where both ES-based scenarios (SC-5 and SC-6) increase the CAIDI average values, as well as the duration of longest interruptions (i.e., tails of PDFs) for the affected customers only. It can be seen that PV scenarios (SC-3 and SC-4) contribute to a reduction in CAIDI index, which seems preferable, but is ultimately unfeasible. It is explained by the fact that PV does not reduce SAIFI, but it lowers SAIDI index by $17.7 \%$ in SC-3 and $26 \%$ in SC-4, respectively. Comparatively, ES-based scenarios reduce SAIFI much more than SAIDI (Table 5).

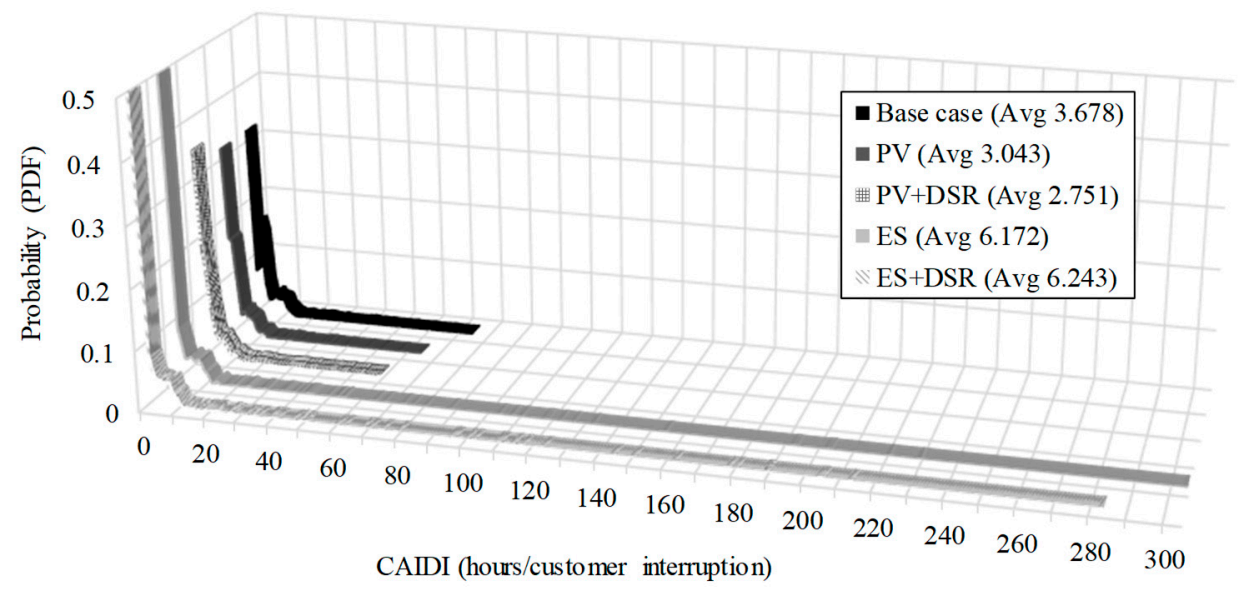

Figure 16. Comparison of PDFs for CAIDI index.

Strict targets set by SQS regulations [7], along with penalty/reward schemes based on network reliability performance, incentivise DNOs to invest in operational tools to ensure lower frequency and duration of supply interruptions. In the UK, for a meshed urban network (as in Figure 3), depending on the interrupted bulk-supplied group demands (GD), different time limits are established to restore supply after the first circuit outage, e.g., 15 mins for GD $\geq 12 \mathrm{MW}, 3 \mathrm{~h}$ for GD $\geq 1 \mathrm{MW}$, or $12 \mathrm{~h}$ (regulator-imposed) to protect customers from excessive LI events [6]. Therefore, the results from the presented reliability assessment methodology demonstrate the benefits of the considered smart grid functionalities and interventions, and their commercial and technical value in terms of improved system and customer reliability performance.

\subsection{Impact on Energy Not Supplied}

Regarding the risk associated with the energy not supplied to customers, Table 6 presents the results for ENS and ACCI indices. As expected, SC-6 results in the most significant reduction to ENS $(41.8 \%)$, because ES is applied as a controlled reliability-corrective action, additionally enhanced by a reliability-oriented DSR scheme. Uncontrolled PV, in combination with DSR, not only reduces the average ENS (by $24.4 \%$ ), but also positively affects the average ENS for the customers experiencing supply interruptions, i.e. ACCI index, reducing it by $16.6 \%$ and $24 \%$ in SC-3 and SC-4, respectively. Accordingly, PV has no net effect on the average number of load points affected by interruptions, as it is locally uncontrolled and therefore cannot prevent the occurrence of upstream network faults. Since PV provides additional energy during faults, it can reduce the average ENS per affected customer. It is important to note that the percentage increases of ACCI, of $64.5 \%$ and $64.2 \%$ in SC-5 and SC- 6 , respectively, should not be interpreted as a weakness of the EMS-controlled ES technology. The reason for this 'increase', as applied in the presented analysis, is the net effect from the ES technology is to greatly reduce the number of interruptions - in most cases, ensuring continuous supply. This means that the total number of affected customers reduce so greatly that the denominator for the calculation of ACCI renders the resulting value higher than in the base case. Implementation of ES in SC-5 and SC-6 is therefore especially efficient in reducing the ACCI, as it also lowers the number of affected customers 
(by over 70\% in both ES-based scenarios). This is deemed to be of great importance to stakeholders, such as DNOs and especially customers who require highly reliable and continuous supply.

Table 6. Energy not supplied and customer curtailment indices.

\begin{tabular}{cccccccc}
\hline ID & Scenario & $\begin{array}{c}\text { ENS } \\
\mathbf{( k W h} / \mathbf{c} / \mathbf{y})\end{array}$ & $*$ & $\begin{array}{c}\text { ACCI } \\
\mathbf{( k W h / A f f e c t e d} \\
\text { Customer) }\end{array}$ & $*$ & $\begin{array}{c}\text { Load Points } \\
\text { Affected (avg) }\end{array}$ & $*$ \\
\hline SC-1 & Base case & 146.37 & - & 1090.41 & - & 6.644 & - \\
SC-3 & PV & 121.90 & $16.7 \%$ & 909.75 & $16.6 \%$ & 6.644 & $0 \%$ \\
SC-4 & PV + DSR & 110.63 & $24.4 \%$ & 828.99 & $24.0 \%$ & 6.644 & $0 \%$ \\
SC-5 & ES & 93.03 & $36.4 \%$ & 1793.87 & $-64.5 \%$ & 1.841 & $72.3 \%$ \\
SC-6 & ES + DSR & 85.21 & $41.8 \%$ & 1790.79 & $-64.2 \%$ & 1.643 & $75.3 \%$ \\
\hline
\end{tabular}

* Reduction from Base Case; $\mathrm{c} / \mathrm{y}=$ customer/year.

\subsubsection{Average Energy Not Supplied}

A significant improvement in average ENS values for different load supply points in the test network, especially when SC-6 is implemented, is further illustrated in Figure 17. The resulting average ENS values exhibit a repetitive pattern from one MV feeder to the next (each with 8 load supply points) due to the symmetry of the network design (Figure 3).

Furthermore, the use of time-varying failure rates and repair times for network PCs, incorporated in the PDFs used as inputs in the MCS algorithm, ensure that the 'randomness' of network behaviour is more accurately modelled. For example, in Figure 17 ES has a higher impact on the ENS reduction at some load supply points, e.g., 27 and 41 on feeders 3 and 6, respectively (over $80 \%$ reduction), than it does at other load supply points, such as 37 and 38 on feeder 5 (approximately 20\% reduction). Overall, ES contributes much more to ENS reduction than DSR, as it represents a post-fault corrective action, while DSR is deployed as a preventive measure to reduce the ENS during the high fault probability periods.

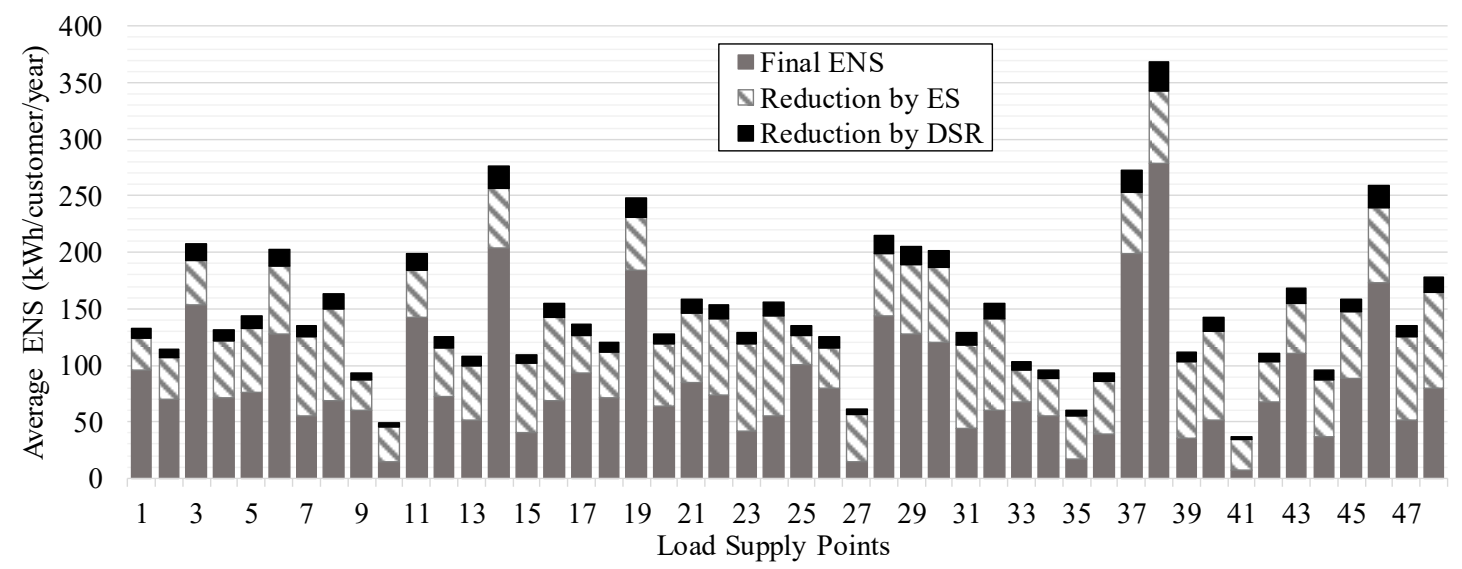

Figure 17. Average ENS for different load supply points.

\subsubsection{Average Customer Curtailment}

Figure 18 illustrates the positive effects of ES + DSR by assessing the probability of different amounts of energy not supplied per interrupted customer (ACCI). It can be seen that SC-6 greatly improves the probability of having no energy curtailment to nearly 0.5 , from 0.32 in the base case, which is directly related to the enhancement of reliability performance and continuity of supply. However, an important feature in Figure 18 is the higher probability of larger values of ENS ( $>4000 \mathrm{kWh} /$ customer interrupted) when ES + DSR is deployed, as compared to values from the uncontrolled PV cases, or the base case. Although it is seemingly a weakness, this is explained by the fact that ES effectively prevents occurrence of system interruptions by providing a back-up continuous 
microgrid-based supply to the customers. For the calculation of ACCI, this means relatively short sustained interruptions will be "converted" to continuous supply, based on the available SOC of the ES, rendering load points with these DERs no longer affected by the upstream faults. However, this is not the case for the relatively longer sustained interruptions, resulting in a lower number of affected customers, but relatively unchanged individual interruption durations and energy unsupplied. This may be clarified by using an example from the presented results, where for one particular year (before any smart intervention-base case), there were eight affected load points, with one relatively long LI and seven relatively short LIs. After the application of ES, the seven relatively short LIs were converted to continuous supply, but one relatively long LI was largely the same, somewhat reducing only the ENS for that period. This results in a much higher ACCI for this particular year when ES is deployed, due to the modified (i.e. reduced) number of customers affected, as compared to the base case. Therefore, it is important to emphasise that ES is undoubtedly the most effective DER technology for improving reliability performance, even though this may not be apparent and immediately clear from the presented results for some indices.

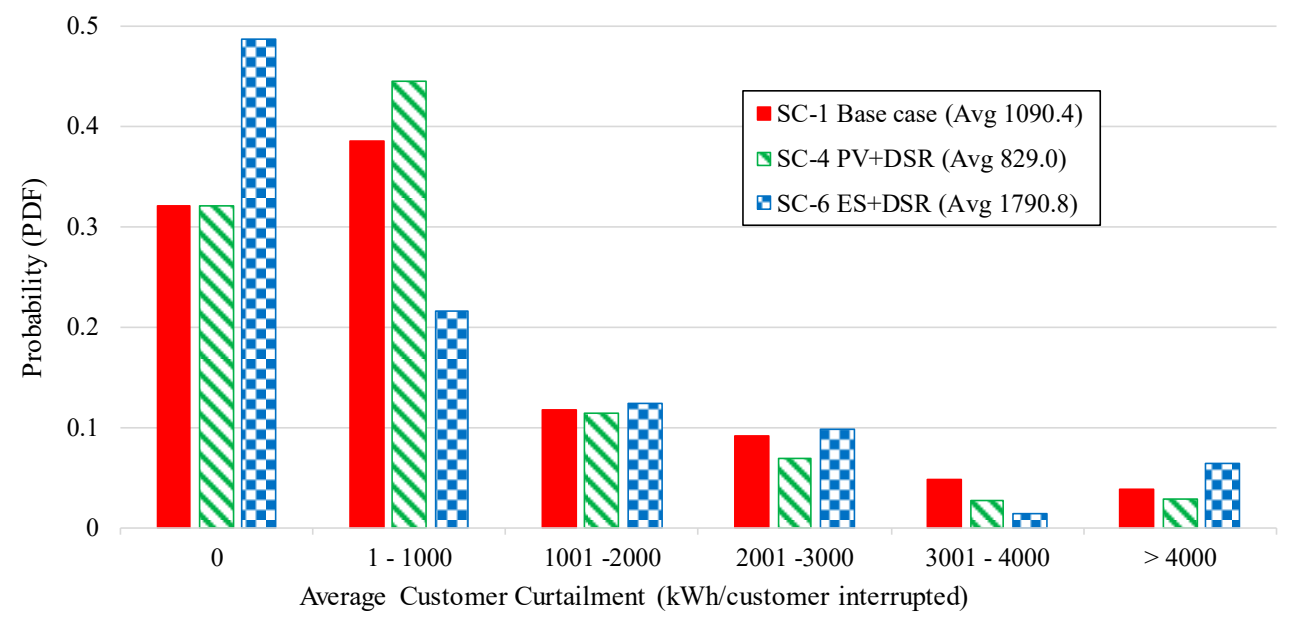

Figure 18. Comparison of PDFs for ACCI values for different scenarios.

\section{Conclusions}

This paper provides an in-depth analysis of the potential reliability performance improvements from the implementation of three smart grid DER functionalities: PV-generation, energy storage (ES) and demand-side response (DSR). The potentials of these technologies are assessed by percentage improvements in the calculated standard reliability indices over the base case (no DERs). The presented methodology is illustrated for a typical meshed underground MV distribution system, in which supplied LV networks are modelled by equivalent aggregate representations. Details of the methodology, modelled urban MV and LV networks, power components data, DER characteristics and all modelling assumptions have been thoroughly described, including the corresponding deployment of the proposed DER scenarios (SC-2 to SC-6). The key benefits of the proposed methodology are in its wider applicability to varying system configurations and load mixes, as well as its capability to demonstrate the relative reliability-performance benefits of different DER technologies. However, it is important to note that when assessing very large systems, i.e. combining multiple MV/LV networks or even different energy vectors, this method is only limited in efficacy by the computational time and burden required, as well as the aggregation techniques to lower system complexity. For that purpose, specifically tailored aggregation techniques should be integrated within this methodology, which will form part of further work by the authors.

A detailed analysis of the presented results provides valuable information as to how to address the stochastic behaviour of PV generation, in order to optimise network reliability performance. It is shown that using an intelligent energy management system (EMS) that controls operation of an ES installation, 
while taking into account variations in state of charge levels of ES, significantly improves network reliability performance through the reductions of frequency and duration of interruptions, as well as energy not supplied to the customers (ENS). In analysing PV-based generation, the paper addresses the impact of cloud movements and transients on the solar PV power output, the associated energy loss and the effects of these on system reliability performance. It is generally found that overestimation of the benefits offered by PV (over 22\%) is possible if the effects of clouding are not accurately accounted for in the PV models. Results demonstrate the capability of ES to be especially efficient in reducing the number of interrupted customers, and more importantly, the energy not supplied to affected customers. The presented work provides contributions in terms of developing more flexible and more accurate reliability assessment tools and improved techniques that can be easily deployable in real-life applications for the evaluation of most cost-effective DER solutions. DNOs can take advantage of such tools for use in system planning operations whereby relative reliability performance benefits, tied to the deployment of various DERs, can be justified both technically and financially.

Author Contributions: Writing-original draft preparation, simulations, software, M.B.N.; formal analysis, supervision, validation, data curation, writing - review and editing, I.H.-G.; conceptualisation, visualisation and resources, S.Z.D. and I.H.G.

Acknowledgments: The authors wish to thank the reviewers for their helpful and constructive comments.

Conflicts of Interest: The authors declare no conflicts of interest.

\section{References}

1. FERC. Title XIII: Smart Grid. In Energy Independence and Security Act of 2007; 110th United States Congress: Washington, DC, USA, 2007.

2. Hernando-Gil, I.; Ilie, I.S.; Collin, A.J.; Acosta, J.L.; Djokic, S.Z. Impact of DG and energy storage on distribution network reliability: A comparative analysis. In Proceedings of the 2012 IEEE International Energy Conference and Exhibition (ENERGYCON), Florence, Italy, 9-12 September 2012.

3. NationalGridESO. Balancing Services. Available online: https://www.nationalgrideso.com/balancingservices (accessed on 10 October 2018).

4. Ndawula, M.B.; Hernando-Gil, I.; Djokic, S. Impact of the stochastic behaviour of distributed energy resources on MV/LV network reliability. In Proceedings of the 2018 IEEE International Conference on Environment and Electrical Engineering and 2018 IEEE EEEIC/I\&CPS Europe), Palermo, Italy, 12-15 June 2018; pp. 1-6.

5. Wang, Y.; Lin, X.; Pedram, M. Adaptive control for energy storage systems in households with photovoltaic modules. IEEE Trans. Smart Grid 2014, 5, 992-1001. [CrossRef]

6. OFGEM. The Electricity (Connection Standards of Performance) Regulations; OFGEM: London, UK, 2015.

7. ENA. Engineering Recommendation p2-6, Security of Supply; Energy Networks Association (ENA); ENA: London, UK, 2005.

8. IEEE Guide for Electric Power Distribution Reliability Indices; IEEE Std 1366-2003; IEEE: Washington, DC, USA, 2004; pp. 1-50.

9. Ndawula, M.B.; Zhao, P.; Hernando-Gil, I. Smart application of energy management systems for distribution network reliability enhancement. In Proceedings of the 2018 IEEE International Conference on Environment and Electrical Engineering and 2018 IEEE EEEIC/I\&CPS Europe, Palermo, Italy, 12-15 June 2018; pp. 1-5.

10. Alam, M.J.E.; Muttaqi, K.M.; Sutanto, D. A novel approach for ramp-rate control of solar PV using energy storage to mitigate output fluctuations caused by cloud passing. IEEE Trans. Energy Convers. 2014, 29, 507-518.

11. Bass, R.B.; Carr, J.; Aguilar, J.; Whitener, K. Determining the power and energy capacities of a battery energy storage system to accommodate high photovoltaic penetration on a distribution feeder. IEEE Power Energy Technol. Syst. J. 2016, 3, 119-127. [CrossRef]

12. Faza, A. A probabilistic model for estimating the effects of photovoltaic sources on the power systems reliability. Reliab. Eng. Syst. Saf. 2018, 171, 67-77. [CrossRef]

13. Adefarati, T.; Bansal, R.C. Reliability, economic and environmental analysis of a microgrid system in the presence of renewable energy resources. Appl. Energy 2019, 236, 1089-1114. [CrossRef] 
14. Sa'ed, J.; Favuzza, S.; Massaro, F.; Telaretti, E. Optimization of bess capacity under a peak load shaving strategy. In Proceedings of the 2018 IEEE International Conference on Environment and Electrical Engineering and 2018 IEEE Industrial and Commercial Power Systems Europe (EEEIC/I\&CPS Europe), Palermo, Italy, 12-15 June 2018; pp. 1-4.

15. Zheng, Y.; Meng, K.; Luo, F.; Qiu, J.; Zhao, J. Optimal integration of MBESSS/SBESSS in distribution systems with renewables. IET Renew. Power Gener. 2018, 12, 1172-1179. [CrossRef]

16. Qiao, Z.; Yang, J. Comparison of centralised and distributed battery energy storage systems in LV distribution networks on operational optimisation and financial benefits. J. Eng. 2017, 2017, 1671-1675. [CrossRef]

17. Mohamad, F.; Teh, J. Impacts of energy storage system on power system reliability: A systematic review. Energies 2018, 11, 1749. [CrossRef]

18. Hernando-Gil, I.; Hayes, B.; Collin, A.; Djokić, S. Distribution network equivalents for reliability analysis. Part 1: Aggregation methodology. In Proceedings of the IEEE PES ISGT Europe 2013, Lyngby, Denmark, 6-9 October 2013; pp. 1-5.

19. Jasmon, G.B.; Lee, L.H.C.C. Distribution network reduction for voltage stability analysis and loadflow calculations. Int. J. Electr. Power Energy Syst. 1991, 13, 9-13. [CrossRef]

20. Trichakis, P.; Taylor, P.C.; Lyons, P.F.; Hair, R. Predicting the technical impacts of high levels of small-scale embedded generators on low-voltage networks. IET Renew. Power Gener. 2008, 2, 249-262. [CrossRef]

21. Billinton, R.; Allan, R.N. Reliability Evaluation of Power Systems; Springer: Boston, MA, USA, 1984.

22. Billinton, R.; Sankarakrishnan, A. A comparison of monte carlo simulation techniques for composite power system reliability assessment. In Proceedings of the IEEE WESCANEX 95. Communications, Power, and Computing. Conference Proceedings, Winnipeg, MB, Canada, 15-16 May 1995; Volume 141, pp. 145-150.

23. Billinton, R.; Jonnavithula, A. Application of sequential monte carlo simulation to evaluation of distributions of composite system indices. IEE Proc. Gener. Transm. Distrib. 1997, 144, 87-90. [CrossRef]

24. Hernando-Gil, I.; Ilie, I.S.; Djokic, S.Z. Reliability planning of active distribution systems incorporating regulator requirements and network-reliability equivalents. IET Gener. Transm. Distrib. 2016, 10, 93-106. [CrossRef]

25. Hernando Gil, I. Integrated Assessment of Quality of Supply in Future Electricity Networks; The University of Edinburgh: Edinburgh, Scotland, UK, 2014.

26. Ilie, I.; Hernando-Gil, I.; Djokic, S.Z. Reliability equivalents of LV and MV distribution networks. In Proceedings of the 2012 IEEE International Energy Conference and Exhibition (ENERGYCON), Florence, Italy, 9-12 September 2012; pp. 343-348.

27. Hadjsaïd, N.; Sabonnadière, J.C. Electrical Distribution Networks; Wiley: Hoboken, NJ, USA, 2013.

28. Martinez-Velasco, A.J.; Guerra, G. Reliability analysis of distribution systems with photovoltaic generation using a power flow simulator and a parallel monte carlo approach. Energies 2016, 9, 537. [CrossRef]

29. Hernando-Gil, I.; Ilie, I.S.; Djokic, S.Z. Reliability performance of smart grids with demand-side management and distributed generation/storage technologies. In Proceedings of the 2012 3rd IEEE PES Innovative Smart Grid Technologies Europe (ISGT Europe), Berlin, Germany, 14-17 October 2012; pp. 1-8.

30. Hernando-Gil, I.; Hayes, B.; Collin, A.; Djokić, S. Distribution network equivalents for reliability analysis. Part 2: Storage and demand-side resources. In Proceedings of the IEEE PES ISGT Europe 2013, Lyngby, Denmark, 6-9 October 2013; pp. 1-5.

31. High Penetration of PV Systems into the Distribution Grid; U.S. Department of Energy: Washing, DC, USA, 2009.

32. Mather, B.; Neal, R. Integrating high penetrations of PV into southern california: Year 2 project update. In Proceedings of the 2012 38th IEEE Photovoltaic Specialists Conference, Austin, TX, USA, 3-8 June 2012; pp. 000737-000741.

33. Smith, J.W.; Dugan, R.; Sunderman, W. Distribution modeling and analysis of high penetration PV. In Proceedings of the 2011 IEEE Power and Energy Society General Meeting, Detroit, MI, USA, 24-29 July 2011; pp. 1-7.

34. Smith, J.W.; Dugan, R.; Rylander, M.; Key, T. Advanced distribution planning tools for high penetration PV deployment. In Proceedings of the 2012 IEEE Power and Energy Society General Meeting, San Diego, CA, USA, 22-26 July 2012; pp. 1-7.

35. Chang, G.W.; Chen, Y.H.; Hsu, L.Y.; Chen, Y.Y.; Chang, Y.R.; Lee, Y.D. Study of impact on high PV-penetrated feeder voltage due to moving cloud shadows. In Proceedings of the 2016 International Symposium on Computer, Consumer and Control (IS3C), Xi'an, China, 4-6 July 2016; pp. 1067-1070. 
36. Graabak, I.; Korpås, M. Variability characteristics of european wind and solar power resources-A review. Energies 2016, 9, 449. [CrossRef]

37. Zhang, D.; Guo, J.; Li, J. Coordinated control strategy of hybrid energy storage to improve accommodating ability of PV. J. Eng. 2017, 2017, 1555-1559. [CrossRef]

38. ENA. Engineering Recommendation g83 Energy Networks Association (ENA); ENA: London, UK, 2018.

39. Zhao, P.; Hernando-Gil, I.; Wu, H. Optimal energy operation and scalability assessment of microgrids for residential services. In Proceedings of the 2018 IEEE International Conference on Environment and Electrical Engineering and 2018 IEEE Industrial and Commercial Power Systems Europe (EEEIC), Palermo, Italy, 12-15 June 2018; pp. 1-6.

40. Lili, C.; Longhua, M.; Zhong, L. Analysis of the operating characteristics of a PV-diesel-BESS microgrid system. Power Syst. Prot. Control 2015, 43, 86-91.

41. Wang, F.; Zhou, L.; Ren, H.; Liu, X.; Talari, S.; Shafie-khah, M.; Catalão, J.P.S. Multi-objective optimization model of source-load-storage synergetic dispatch for a building energy management system based on tou price demand response. IEEE Trans. Ind. Appl. 2018, 54, 1017-1028. [CrossRef]

42. Zhao, P.; Wu, H.; Hernando-Gil, I. Optimal home energy management under hybrid PV-storage uncertainty: A distributionally robust chance constrained approach. IET Renew. Power Gener. 2018. Submitted.

43. Zhao, C.; Dong, S.; Gu, C.; Li, F.; Song, Y.; Padhy, N.P. New problem formulation for optimal demand side response in hybrid ac/dc systems. IEEE Trans. Smart Grid 2018, 9, 3154-3165. [CrossRef]

44. TrinaSolar. TSM_PD14 Datasheet B. Product Datasheet; TrinaSolar: San Jose, CA, USA, 2017.

45. McDermott, T.E.; Dugan, R.C. Distributed generation impact on reliability and power quality indices. In Proceedings of the 2002 Rural Electric Power Conference, Papers Presented at the 46th Annual Conference (Cat. No. 02CH37360), Colorado Springs, CO, USA, 5-7 May 2002.

(C) 2019 by the authors. Licensee MDPI, Basel, Switzerland. This article is an open access article distributed under the terms and conditions of the Creative Commons Attribution (CC BY) license (http:/ / creativecommons.org/licenses/by/4.0/). 